\title{
Axial vector diquark correlations in the nucleon: \\ Structure functions and static properties *
}

\author{
H. Mineo \\ Department of Physics, Faculty of Science, University of Tokyo \\ Bunkyo-ku, Hongo, Tokyo 113-0033, Japan \\ W. Bentz \\ Department of Physics, School of Science, Tokai University \\ Hiratsuka-shi, Kanagawa 259-1292, Japan \\ N. Ishii \\ The Institute of Physical and Chemical Research (RIKEN) \\ Hirosawa, Wako-shi, Saitama 351-0198, Japan \\ K. Yazaki \\ Department of Physics, Faculty of Humanities and Sciences, \\ Tokyo Woman's Christian University \\ Suginami-ku, Tokyo 167-8585, Japan
}

${ }^{*}$ Correspondence to: H. Mineo, E-mail: mineo@nt.phys.s.u- tokyo.ac.jp 


\begin{abstract}
In order to extract information on the strength of quark-quark correlations in the axial vector (a.v.) diquark channel $\left(J^{P}=1^{+}, T=1\right)$, we analyze the quark light cone momentum distributions in the nucleon, in particular their flavor dependencies, and the static properties of the nucleon. To construct the nucleon as a relativistic 3-quark bound state, we use a simple 'static' approximation to the full Faddeev equation in the Nambu-Jona-Lasinio model, including correlations in the scalar $\left(J^{P}=0^{+}, T=0\right)$ and a.v. diquark channels. It is shown that the a.v. diquark correlations should be rather weak compared to the scalar ones. From our analysis we extract information on the strength of the correlations as well as on the probability of the a.v. diquark channel.
\end{abstract}

PACS numbers: 12.39-x, 12.39.Ki, 14.20.Dh

Keywords: Structure functions, Effective quark theories, Diquark correlations 


\section{Introduction}

The investigation of nucleon structure functions and the study of the associated quark and gluon light cone (LC) momentum and spin distributions is currently a very active field both experimentally [1, 2, 3] and theoretically [四]. By applying perturbative QCD and factorization theorems [5] to the analysis of deep inelastic lepton-nucleon scattering experiments, detailed information on the quark and gluon distributions in the nucleon have been (and will further be) extracted. Many excellent parameterizations of quark and gluon distributions now exist [6]. Since these distributions reflect the nonperturbative aspects of the problem, at present they cannot be calculated directly from QCD. Effective models based on QCD are powerful tools to extract information on the effective quark-quark interactions by comparing the calculated distributions with the empirical ones.

In this paper we will concentrate on the quark LC momentum distributions relevant for the unpolarized structure functions. A particularly interesting feature of the valence $(v)$ quark distributions in the proton is their flavor dependence: The empirical distributions clearly indicate that $d_{v}(x)$ is "softer" than $u_{v}(x)$, i.e., it is more concentrated in the region of smaller Bjorken $x$. There is a very simple argument [7] which shows that this feature can be naturally explained as a consequence of the quark-quark (diquark) correlations in the scalar $\left(J^{P}=0^{+}, T=0\right)$ channel: Strong correlations in this channel lead to a "scalar diquark" with mass well below twice the constituent quark mass. Since the fraction of the LC momentum carried by the diquark decreases as its mass decreases, and a scalar diquark consists of a valence up and down quark, this means that $d_{v}$ in the proton is softer than 
$u_{v}$ for sufficiently strong scalar diquark correlations.

However, the correlations in the axial vector (a.v.) diquark channel $\left(J^{P}=1^{+}, T=1\right)$ are expected to be of some importance, too, from the analogy of non- relativistic 3-body calculations. Compared to a purely quark - scalar diquark model, these correlations tend to work towards more symmetric valence quark distributions, since their presence requires a reduction of the correlations in the scalar diquark channel in order to get the same nucleon mass. Indeed, the ratio $F_{2 n} / F_{2 p}$ of the neutron and proton structure functions in the region $x \rightarrow 1$, where the valence quarks are dominant, indicates [8] that $d_{v} / u_{v}$ is probably nonzero (but less than the value $\frac{1}{2}$ corresponding to flavor symmetric distributions) as $x \rightarrow 1$, although there is some model dependence in extracting $F_{2 n}$ from the deuteron data. One of the questions which we want to address in this paper is therefore how the flavor asymmetry of the valence quark distributions is influenced by the a.v. diquark correlations.

Another interesting feature of the distribution functions is the flavor asymmetry of the antiquark distributions in the proton [9], i.e., $\bar{d}(x)>\bar{u}(x)$ over the whole region of Bjorken $x$, which leads to the famous violation of the Gottfried sum rule. A simple and natural explanation of this asymmetry is due to the pion cloud around the valence quarks, and there have been many investigations of this effect based on the familiar convolution formalism [10].

The primary aim of this paper is to investigate the connection between the flavor dependence of the valence quark distributions and the strength of the quark-quark interactions in the a.v. diquark channel in a model calculation. For this purpose we use the Nambu-Jona- Lasinio [11] (NJL) model as an effective quark theory in the low energy region [12]. Our approach is based 
on the relativistic Faddeev method [13], which has been used in many recent works as a powerful tool to investigate the properties of hadrons [14, 15, 16]. 1] Although the relativistic Faddeev equation in the NJL model has been solved exactly including also the a.v. diquark channel [14], this has not yet been done with LC variables, mainly due to technical reasons associated with regularization and angular momentum projection [19]. Therefore, in this work we consider a simple approximation to the full Faddeev equation [20] (the "static approximation"), which consists in neglecting the momentum dependence of the quark exchange kernel. In this case, the resulting quark-diquark equation can be solved almost analytically. In a previous work we applied this method to the calculation of structure functions by keeping only the scalar diquark channel [21], and here we extend these calculations to include also the a.v. diquark channel. We will derive an upper limit for the strength of the quark-quark interaction in this channel, which can be translated into an upper limit of the probability (defined here via the contribution to the baryon number). In order to see whether this result derived from the flavor dependence of the structure functions is consistent with other properties of the nucleon or not, we will also investigate the dependence of static properties, like magnetic moments or the axial vector coupling constant, on the correlations in the a.v. diquark channel. We will show that a consistent picture emerges if the probability of the a.v. diquark channel is $\lesssim 10 \%$, although the model has still to be refined for more quantitative purposes.

The rest of this paper is organized as follows: In sect. 2 we explain the model and the form of the nucleon wave function including the a.v.

\footnotetext{
${ }^{1}$ Very similar in spirit are the covariant quark-diquark models [17, 18], which have been extensively used recently to describe many properties of the nucleon like electromagnetic form factors and static properties.
} 
diquark channel. In sect. 3 we explain our method to calculate the quark distribution functions, and in sect. 4 we consider the static properties of the nucleon within the same framework. Sect. 5 is devoted to the discussion of our results, and in sect. 6 we present a summary and conclusions.

\section{The model and vertex functions for baryon states}

We consider $\mathrm{SU}(2)_{f}$ quark Lagrangians of the NJL [1] type $\mathcal{L}=\bar{\psi}(\not \not \supset-$ $m) \psi+\mathcal{L}_{I}$, where $m$ is the $\mathrm{u}$, d current quark mass and $\mathcal{L}_{I}$ is a chirally symmetric 4-fermi contact interaction. By applying Fierz transformations, any $\mathcal{L}_{I}$ can be decomposed into various $q \bar{q}$ and $q q$ channels 14 . The terms relevant for our discussions are given as follows:

$$
\begin{aligned}
\mathcal{L}_{I, \pi} & =\frac{1}{2} G_{\pi}\left[(\bar{\psi} \psi)^{2}-\left(\bar{\psi} \gamma_{5} \boldsymbol{\tau} \psi\right)^{2}\right] \\
\mathcal{L}_{I, s} & =G_{s}\left[\bar{\psi}\left(\gamma_{5} C\right) \tau_{2} \beta^{A} \bar{\psi}^{T}\right]\left[\psi^{T}\left(C^{-1} \gamma_{5}\right) \tau_{2} \beta^{A} \psi\right] \\
\mathcal{L}_{I, a} & =G_{a}\left[\bar{\psi}\left(\gamma_{\mu} C\right) \boldsymbol{\tau} \tau_{2} \beta^{A} \bar{\psi}^{T}\right] \cdot\left[\psi^{T}\left(C^{-1} \gamma_{\mu}\right) \boldsymbol{\tau} \tau_{2} \beta^{A} \psi\right]
\end{aligned}
$$

where the color matrix $\beta^{A}=\sqrt{\frac{3}{2}} \lambda^{A}(\mathrm{~A}=2,5,7)$ corresponds to the color $\overline{3}$ states, and $C=i \gamma_{2} \gamma_{0} . \mathcal{L}_{I, \pi}$ represents the interaction in the $0^{+}$and $0^{-} q \bar{q}$ channels corresponding to the sigma meson and the pion, and $\mathcal{L}_{I, s}\left(\mathcal{L}_{I, a}\right)$ is the interaction in the $0^{+}\left(1^{+}\right) q q$ channel corresponding to the scalar diquark (a.v. diquark). The interactions (2.1) and (2.2) are invariant under chiral $\mathrm{SU}(2) \times \mathrm{SU}(2)$ transformations, but $(2.3)$ should in principle be supplemented by the vector diquark $\left(1^{-}\right)$interaction term to form a chiral invariant lagrangian [16]. However, from the naive non-relativistic analogy the vector diquark is expected to be of minor importance since it is a $\ell=1$ pair, and 
will be neglected here.

The coupling constants $G_{\pi}, G_{s}$ and $G_{a}$ are related numerically to the ones of the original $\mathcal{L}_{I}$. Since $G_{\pi}$ will be determined by the properties of the pion, it is convenient to introduce the ratios

$$
r_{s}=G_{s} / G_{\pi}, \quad r_{a}=G_{a} / G_{\pi} .
$$

These ratios will be treated as parameters reflecting the form of the original $\mathcal{L}_{I}$. (For example, for the 'color current' type interaction lagrangian [20] one has $r_{s}=0.5$ and $r_{a}=0.25$.)

The reduced t-matrices in the pionic $q \bar{q}$ channel, and the scalar and axial vector $q q$ channels are obtained by solving the Bethe-Salpeter equations in these channels, and the results are the following standard NJL model expressions [11, [12]:

$$
\begin{array}{r}
\tau_{\pi}(k)=\frac{-2 i G_{\pi}}{1+2 G_{\pi} \Pi_{\pi}\left(k^{2}\right)}, \quad \tau_{s}(k)=\frac{4 i G_{s}}{1+2 G_{s} \Pi_{s}\left(k^{2}\right)}, \\
\tau_{a}^{\mu \nu}(k)=4 i G_{a}\left[g^{\mu \nu}-\frac{2 G_{a} \Pi_{a}\left(k^{2}\right)}{1+2 G_{a} \Pi_{a}\left(k^{2}\right)}\left(g^{\mu \nu}-\frac{k^{\mu} k^{\nu}}{k^{2}}\right)\right],
\end{array}
$$

with the "bubble graphs"

$$
\begin{array}{r}
\Pi_{\pi}\left(k^{2}\right)=\Pi_{s}\left(k^{2}\right)=6 i \int \frac{d^{4} q}{(2 \pi)^{4}} \operatorname{tr}_{D}\left[\gamma_{5} S(q) \gamma_{5} S(k+q)\right], \\
\Pi_{a}\left(k^{2}\right)\left(g^{\mu \nu}-\frac{k^{\mu} k^{\nu}}{k^{2}}\right)=6 i \int \frac{d^{4} q}{(2 \pi)^{4}} \operatorname{tr}_{D}\left[\gamma^{\mu} S(q) \gamma^{\nu} S(k+q)\right],
\end{array}
$$

where $S(k)=(\not k-M+i \epsilon)^{-1}$ is the quark Feynman propagator and $M$ is the constituent quark mass, which is related to the current quark mass $m$ via the familiar gap equation of the NJL model [11].

For the evaluation of these and other loop integrals we will use the "covariant three-momentum cut-off scheme" [12, 19], by which we mean the 
following: After parameterizing the expressions for the Green functions in terms of Lorentz invariant functions (like $\Pi_{a}$ in eq. (2.8)), these Lorentz invariants are calculated by introducing a sharp three-momentum cut-off $\left(\Lambda_{3}\right)$ in a particular Lorentz frame, which we take to be the rest frame $(\boldsymbol{k}=0)$ in case of two-point functions and the Breit frame in the case of three- point functions involving one vertex for the external field. The Lorentz invariant expressions are then recovered by "boosting" to a general frame. (In the case of the above bubble graphs, this "boosting" simply means the replacement $k_{0}^{2} \rightarrow k^{2}$.) The value of $\Lambda_{3}$ is assumed to be the same for all loop integrals. The three-momentum cut-off scheme used here preserves the conservation of electric charge and baryon number, but not the full current conservation which involves finite momentum transfer by the external field. It also preserves low energy theorems based on chiral symmetry, but not the full PCAC and Goldberger-Treiman relation (see sect. 5.2).

In order to obtain the nucleon vertex function, we solve the homogeneous Faddeev equation in the "static approximation" [20, 21], where the momentum dependence of the quark exchange kernel $(Z)$ is neglected. Performing the projections to color singlet and isospin $\frac{1}{2}$ as in [14], the resulting equation for the baryon vertex function $\Gamma(p)$ becomes

$$
\Gamma^{a}(p)=Z^{a a^{\prime}} \Pi_{N}^{a^{\prime} b}(p) \Gamma^{b}(p) \equiv K^{a b}(p) \Gamma^{b}(p),
$$

where $Z$ is the quark exchange kernel in the static approximation,

$$
Z^{a a^{\prime}}=\frac{3}{M}\left(\begin{array}{cc}
1 & \sqrt{3} \gamma^{\mu^{\prime}} \gamma_{5} \\
\sqrt{3} \gamma_{5} \gamma^{\mu} & -\gamma^{\mu^{\prime}} \gamma^{\mu}
\end{array}\right),
$$

and $\Pi_{N}(p)$ is the quark-diquark bubble graph

$$
\Pi_{N}^{a^{\prime} b}(p)=\int \frac{d^{4} k}{(2 \pi)^{4}} \tau^{a^{\prime} b}(k) S(p-k)
$$




$$
\tau^{a^{\prime} b}(k)=\left(\begin{array}{cc}
\tau_{s}(k) & 0 \\
0 & \tau_{a}^{\mu^{\prime} \nu}(k)
\end{array}\right) \equiv \operatorname{diag}\left(\tau_{s}(k), \tau_{a}^{\mu^{\prime} \nu}(k)\right) .
$$

In the above equations, roman indices $\left(a, a^{\prime}, \ldots\right)$ combine one index for the scalar diquark (denoted as 5 ) with the 4 Lorentz indices $\left(\mu, \mu^{\prime}, \ldots\right)$ for the a.v. diquark. Together with the Dirac index for the quark (not shown explicitly), the kernel $K$ in eq.(2.9) is a $20 \times 20$ matrix, which can be reduced to $10 \times$ 10 by projection to positive parity states (see Appendix A). We directly diagonalize this kernel in the rest frame of the baryon $(\boldsymbol{p}=0)$ by unitary transformations in Appendix A. It separates into a $6 \times 6$ block corresponding to spin $\frac{1}{2}$ states (nucleon) \& and a $4 \times 4$ block for the spin $\frac{3}{2}$ states. After boosting the eigenfunction to a general frame, we obtain for the nucleon state $\Gamma_{N}^{a}=\left(\Gamma_{N}^{5}, \Gamma_{N}^{\mu}\right)$, where

$$
\begin{aligned}
\Gamma_{N}^{5}(p, s) & =\alpha_{1} u_{N}(p, s) \\
\Gamma_{N}^{\mu}(p, s) & =\alpha_{2} \frac{p^{\mu}}{M_{N}} \gamma_{5} u_{N}(p, s)+\alpha_{3} \sum_{\lambda s^{\prime}}\left(1 \frac{1}{2}, \lambda s^{\prime} \mid \frac{1}{2} s\right) \epsilon_{\lambda}^{\mu}(p) u_{N}\left(p, s^{\prime}\right)
\end{aligned}
$$

Here $u_{N}(p, s)$ is a Dirac spinor, with spin projection $s$, depending on the nucleon mass $M_{N}$ and normalized according to $\bar{u}_{N} u_{N}=1$, and $\epsilon_{\lambda}^{\mu}(p)$ is the polarization 4-vector, which also depends on $M_{N}$, and is obtained as usual by applying a Lorentz boost to $\left(0, \boldsymbol{\epsilon}_{\lambda}\right)$, where $\boldsymbol{\epsilon}_{\lambda}$ are the spherical unit vectors with $\lambda= \pm 1,0$. The homogeneous equation for the 3 coefficients $\alpha_{1}, \alpha_{2}, \alpha_{3}$ leads to the eigenvalue equation for the nucleon mass $M_{N}$ (see Appendix A). Note that in the last term of (2.14) the spin projection $s^{\prime}$ in the Dirac spinor refers to the quark. The vertex function for spin $\frac{3}{2}$ is given by an expression

\footnotetext{
${ }^{2}$ The corresponding 6 basis states correspond to the coupling of the quark with a scalar diquark, with the time component of the a.v. diquark, and with the space components of the a.v. diquark, where each of these states has spin degeneracy 2.
} 
similar to the last term in (2.14), but with the diquark and quark spins coupled to $\frac{3}{2}$ instead of $\frac{1}{2}$. This vertex function is therefore of the standard Rarita-Schwinger form.

By simple manipulations explained in Appendix A, eq.(2.14) can be shown to be equivalent to the following covariant form:

$$
\Gamma_{N}^{\mu}(p, s)=a_{2} \frac{p^{\mu}}{M_{N}} \gamma_{5} u_{N}(p, s)+a_{3} \gamma^{\mu} \gamma_{5} u_{N}(p, s)
$$

with $a_{2}=\alpha_{2}-\alpha_{3} / \sqrt{3}$ and $a_{3}=-\alpha_{3} / \sqrt{3}$. The form (2.15), which is a special case of the general covariant decomposition of the baryon vertex functions given in ref. [17, is more convenient for actual Feynman diagram calculations 円.

The normalization condition for the nucleon vertex function, which we give here in terms of LC variables $p_{ \pm}=(1 / \sqrt{2})\left(p_{0} \pm p_{3}\right)$ for later use, is as follows 22, 19]:

$$
\frac{1}{2 p_{-}} \bar{\Gamma}_{N}^{a}(p) \frac{\partial \prod_{N, a b}(p)}{\partial p_{+}} \Gamma_{N}^{b}(p)=1 \equiv W_{s}+W_{a} .
$$

Here we introduced the "weights" of the scalar and a.v. diquark channels, where $W_{s}$ is the contribution from $a=b=5$ and $W_{a}$ is the remaining part. In any treatment which preserved the Ward identities, the l.h.s. of $(2.16)$ corresponds to the matrix element of the baryon number operator [21], and therefore $W_{s}$ and $W_{a}$ are the contributions of the scalar and a.v. diquark channels to the total baryon number.

\footnotetext{
${ }^{3}$ The isospin and color parts of the vertex functions are standard and not shown explicitly here. We also note that it is of course possible to start directly from the covariant parameterization (2.15), together with the Rarita-Schwinger type part corresponding to spin $\frac{3}{2}$, introduce a covariant decomposition of the kernel in (2.9) and derive the homegenous equations for the coefficients $a_{1} \equiv \alpha_{1}, a_{2}$ and $a_{3}$.
} 
Similarly, the vertex function for the $\Delta$ isobar state is obtained from an eigenvalue equation similar to (2.9) with the kernel projected to color $\overline{3}$ and $T=\frac{3}{2}:$

$$
K^{\mu \nu}(p)=\frac{6}{M}\left(\gamma^{\mu} \gamma_{\rho}\right) \Pi_{N}^{\rho \nu}(p)
$$

with the same quark-diquark bubble $\Pi_{N}(p)$ graph as before (see eq. (2.11)). The corresponding eigenfunction, which has the same form as the last term in eq. (2.14) but with the nucleon mass replaced by the delta mass $M_{\Delta}$ and the total spin $\frac{1}{2}$ replaced by $\frac{3}{2}$ in the Clebsch-Gordan coefficient, is of the standard Rarita-Schwinger form. The eigenvalue equation for the $M_{\Delta}$ is also given in Appendix A.

\section{Quark distribution functions}

The quark LC momentum distribution in the proton is defined as [23]

$$
\tilde{f}_{q / P}(x)=\frac{1}{2} \int \frac{d z^{-}}{2 \pi} e^{i p-x z^{-}}\left\langle p\left|T\left(\bar{\psi}_{q}(0) \gamma^{+} \psi_{q}\left(z^{-}\right)\right)\right| p\right\rangle,
$$

where $x$ is the fraction of the proton's LC momentum component $p_{-}$carried by the quark with flavor $q=u, d$, and $\mid p>$ denotes the proton state with momentum $p$. As explained in [19, 21], the evaluation of the distribution (3.1) can be reduced to a straightforward Feynman diagram calculation by noting that it is nothing but the Fourier transform of the quark two-point function in the proton traced with $\gamma^{+}$, where the component $k_{-}$of the quark LC momentum is fixed as $k_{-}=x p_{-}$and the other quark momentum components $\left(k_{+}, \mathbf{k}_{\perp}\right)$ are integrated out. The relevant Feynman diagrams in our quarkdiquark model are shown in Fig. 1, where the operator insertion stands for $\gamma^{+} \delta\left(k_{-}-p_{-} x\right)\left(1 \pm \tau_{z}\right) / 2$ for the $u(d)$ quark distribution. 
The isospin matrix elements are easily evaluated, and the distributions can be expressed as follows 1 :

$$
\begin{aligned}
& f_{U / P}(x)=F_{Q / P}^{s}(x)+\frac{1}{2} F_{Q(D) / P}^{s}(x)+\frac{1}{3} F_{Q / P}^{a}(x)+\frac{5}{6} F_{Q(D) / P}^{a}(x) \\
& f_{D / P}(x)=\frac{1}{2} F_{Q(D) / P}^{s}(x)+\frac{2}{3} F_{Q / P}^{a}(x)+\frac{1}{6} F_{Q(D) / P}^{a}(x) .
\end{aligned}
$$

Here the distribution $F_{Q / P}^{d}(d=s, a)$ corresponds to the first diagram of fig.1, which will be called the "quark diagram" and involves the diquark t- matrix $\tau_{d}$, and $F_{Q(D) / P}^{d}$ to the second diagram ("diquark diagram") with two $\tau_{d}$ 's. To derive their explicit expressions from the Feynman diagrams, one uses the Ward-like identity $S(k) \gamma^{+} S(k)=-\frac{\partial}{\partial k_{+}} S(k)$ for the constituent quark propagator and performs partial integrations w.r.t. $k_{+}$, which is justified since the regularization scheme to be discussed below (Lepage- Brodsky scheme [24]) does not restrict the $k_{+}$integrals which are convergent. In this way we obtain from the 'quark diagram':

$$
F_{Q / P}(x)=\frac{1}{2 p_{-}} \bar{\Gamma}_{N}^{a}(p)\left(\frac{\partial}{\partial p_{+}} \Pi_{N}^{a b}(x, p)\right) \Gamma_{N}^{b}(p) \equiv F_{Q / P}^{s}+F_{Q / P}^{a},
$$

where the quark-diquark bubble for fixed $k_{-}$is given by

$$
\Pi_{N}^{a b}(x, p)=\int \frac{d^{4} k}{(2 \pi)^{4}} \delta\left(x-\frac{k_{-}}{p_{-}}\right) S(k) \tau^{a b}(p-k)=\operatorname{diag}\left(\Pi_{N}^{55}(x, p), \Pi_{N}^{\mu \nu}(x, p)\right)
$$

For the evaluation of the 'diquark diagram' of Fig.1 one also uses the Wardlike identity for the quark propagator on which the insertion is made, and

\footnotetext{
${ }^{4}$ The distribution $(3.1)$ is nonzero in the interval $-1<x<1$, and the physical quark and antiquark distributions are obtained as $f_{q / P}(x)=\tilde{f}_{q / P}(x)$ and $f_{\bar{q} / P}(x)=-\tilde{f}_{q / P}(-x)$ for $0<x<1$. We denote the quark flavor as $Q=U, D$ for the diagrams of Fig.1 to distinguish this 'valence quark picture' from the case including the pion cloud. In writing down the expressions $(3.2)$ and $(3.3)$ we used the fact that there is no mixing between the scalar and a.v. diquark diagrams for the quark distributions.
} 
introduces the fraction of the nucleon's momentum component $p_{-}$carried by the diquark $(y)$ and the fraction of the diquark's momentum component $q_{-}$ carried by the quark inside the diquark $(z)$ via the identity

$$
1=\int \mathrm{d} y \int \mathrm{d} z \delta\left(y-\frac{q_{-}}{p_{-}}\right) \delta\left(z-\frac{k_{-}}{q_{-}}\right) .
$$

In this way we obtain

$$
\begin{aligned}
F_{Q(D) / P}(x) & =\frac{-i}{2 p_{-}} \int_{0}^{1} \mathrm{~d} y \int_{0}^{1} \mathrm{~d} z \int \frac{\mathrm{d}^{4} q}{(2 \pi)^{4}} \delta\left(y-\frac{q_{-}}{p_{-}}\right) \\
& \times \bar{\Gamma}_{N}^{a}(p) S_{F}(p-q)\left(\tau_{a b}(q) \frac{\partial \Pi^{b c}(z, q)}{\partial q_{+}} \tau_{c d}(q)\right) \bar{\Gamma}_{N}^{d}(p),
\end{aligned}
$$

where the quark-quark bubble graph for fixed LC momentum fraction $z$ of one of the quarks is given by $\Pi^{a b}(z, q)=\operatorname{diag}\left(\Pi_{s}\left(z, q^{2}\right), \Pi_{a}^{\mu \nu}(z, q)\right)$ with

$$
\begin{aligned}
\Pi_{s}\left(z, q^{2}\right) & =6 i \int \frac{d^{4} k}{(2 \pi)^{4}} \delta\left(z-\frac{k_{-}}{q_{-}}\right) \operatorname{tr}_{D}\left(\gamma_{5} S(k) \gamma_{5} S(q-k)\right) \\
\Pi_{a}^{\mu \nu}(z, q) & \equiv \Pi_{a}\left(z, q^{2}\right)\left(g^{\mu \nu}-\frac{q^{\mu} q^{\nu}}{q^{2}}\right) \\
& =6 i \int \frac{d^{4} k}{(2 \pi)^{4}} \delta\left(z-\frac{k_{-}}{q_{-}}\right) \operatorname{tr}_{D}\left[\gamma^{\mu} S(k) \gamma^{\nu} S(q-k)\right]
\end{aligned}
$$

Since the quark-quark bubble graphs $\Pi^{a b}(z, q)$ and $\Pi^{a b}(q)$ have the same structure w.r.t. the diquark indices (see (2.8) and (3.8)), we can define the quark LC momentum distribution $F_{Q / D}^{d}\left(z, q^{2}\right)$ within a diquark $d=s, a$ with virtuality $q^{2}$ by

$$
\frac{\partial \Pi^{a b}(z, q)}{\partial q_{+}} \equiv \operatorname{diag}\left(\frac{1}{2} \frac{\partial \prod_{s}\left(q^{2}\right)}{\partial q_{+}} F_{Q / D}^{s}\left(z, q^{2}\right), \frac{1}{2} \frac{\partial \Pi_{a}^{\mu \nu}(q)}{\partial q_{+}} F_{Q / D}^{a}\left(z, q^{2}\right)\right)
$$

By taking the trace over the Lorentz indices in this equation for the a.v. contribution, we find explicitly

$$
F_{Q / D}^{d}\left(z, q^{2}\right)=-2 g_{d}^{2}\left(q^{2}\right) \frac{\partial \Pi_{d}\left(z, q^{2}\right)}{\partial q^{2}}
$$


where

$$
g_{d}\left(q^{2}\right) \equiv \frac{-1}{\frac{\partial \Pi_{d}\left(q^{2}\right)}{\partial q^{2}}}
$$

with $d=s, a$. The quantities (3.11) are the natural generalizations of the quark-diquark coupling constants to arbitrary virtuality $q^{2}$. Inserting the definition (3.9) into (3.6), using the Ward-like identity for the derivatives of quark-quark bubble graphs

$$
\left(\tau_{a b}(q) \frac{\partial \Pi^{b c}(q)}{\partial q_{+}} \tau_{c d}(q)\right)=-2 i \frac{\partial \tau^{a d}(q)}{\partial q_{+}}
$$

and performing a partial integration w.r.t. $q_{+}$we finally arrive at

$$
F_{Q(D) / P}(x)=\int_{0}^{1} \mathrm{~d} y \int_{0}^{1} \mathrm{~d} z \delta(x-y z) \int_{-\infty}^{\infty} \mathrm{d} q_{0}^{2} \sum_{d=s, a} F_{Q / D}^{d}\left(z, q_{0}^{2}\right) F_{D / P}^{d}\left(y, q_{0}^{2}\right)
$$

Here the LC momentum distribution of the diquark $\mathrm{d}$ with virtuality $q_{0}^{2}$ in the proton is given by

$$
\begin{aligned}
F_{D / P}\left(y, q_{0}^{2}\right) & \equiv F_{D / P}^{s}\left(y, q_{0}^{2}\right)+F_{D / P}^{a}\left(y, q_{0}^{2}\right) \\
& =\bar{\Gamma}_{N}^{a}(p)\left(\frac{1}{2 p_{-}} \frac{\partial}{\partial p_{+}}+y \frac{\partial}{\partial q_{0}^{2}}\right) \Pi_{N}^{a b}\left(y, q_{0}^{2} ; p\right) \Gamma_{N}^{b}(p),
\end{aligned}
$$

where the quark-diquark bubble graph for fixed virtuality and LC momentum fraction of the diquark is given by

$$
\Pi_{N}^{a b}\left(y, q_{0}^{2} ; p\right)=\int \frac{d^{4} q}{(2 \pi)^{4}} \delta\left(y-\frac{q_{-}}{p_{-}}\right) \delta\left(q^{2}-q_{0}^{2}\right) S_{F}(p-q) \tau^{a b}(q) .
$$


The normalizations of the distributions $F_{Q / P}(x)$ and $F_{Q(D) / P}(x)$ follow from eqs. (3.4), (3.13) and (2.16) as follows of:

$$
\begin{gathered}
\int_{0}^{1} \mathrm{~d} x F_{Q / P}(x)=W_{s}+W_{a}=1 \\
\int_{0}^{1} \mathrm{~d} x F_{Q(D) / P}(x)=2\left(W_{s}+W_{a}\right)=2,
\end{gathered}
$$

which lead to the correct number sum rules for the distributions (3.2) and (3.3). In the same way the validity of the momentum sum rule can also be checked analytically. These sum rules hold in any regularization scheme which does not restrict the LC plus- components $\left(k_{+}\right)$of the loop momenta [21].

For the evaluation of the quark-quark and quark-diquark bubble graphs with fixed LC momentum fraction of one of the constituents we use the Lepage-Brodsky (LB) regularization scheme [24], which is equivalent to the covariant three-momentum cut-off scheme discussed in the previous subsection if all internal momenta are integrated out 19. Because this scheme does not restrict the LC plus-components of the loop momenta, it preserves the number and momentum sum rules. In practice, to regularize bubble graphs involving Lorentz indices like $\Pi_{a}^{\mu \nu}$ of eq. (3.8) or $\Pi_{N}^{\mu \nu}$ of eq. (3.15), one first decomposes them into a sum of Lorentz tensors multiplied by Lorentz scalar quantities (which, for the case of $\Pi_{N}^{\mu \nu}$, are Dirac matrices $\propto 1$ or $\not p$ ), and then applies the LB scheme to evaluate the scalar functions in the frame where the transverse components of the total momentum are zero. (To extract the scalar functions from the general Lorentz decomposition in this frame, one

\footnotetext{
${ }^{5}$ Note that the relations $\int_{0}^{1} \mathrm{~d} z F_{Q / D}^{d}\left(z, q_{0}^{2}\right)=2$ and $\int_{0}^{1} \mathrm{~d} z z F_{Q / D}^{d}\left(z, q_{0}^{2}\right)=1$ hold for any virtuality $q_{0}^{2}$, and that the second term in (3.14) gives a vanishing surface term when integrated over $q_{0}^{2}$.
} 
considers appropriate combinations of the Lorentz indices.) The results are then generalized to an arbitrary frame in a similar way as explained for the covariant 3-momentum cut-off.

The model described above gives only valence-like distributions at the low energy scale. In order to describe also the sea quark distributions, we take into account the effects of the pion cloud around the constituent quarks in the same way as described in detail in ref. [21]. This treatment corresponds to the standard one-dimensional convolution formalism [10], which involves an on- shell approximation for the "parent quark". The resulting distribution functions including the pion cloud contributions are given by

$$
f_{q / P}(x)=\sum_{Q=U, D} \int_{0}^{1} \mathrm{~d} y \int_{0}^{1} \mathrm{~d} z \delta(x-y z) f_{q / Q}(z) f_{Q / P}(y)
$$

and a similar expression with $q \rightarrow \bar{q}$. The valence- like distributions $f_{Q / P}(y)$ are given by eq. (3.2) and (3.3), and the expressions for the quark and antiquark distributions within an on-shell parent quark $\left(f_{q / Q}\right.$ and $\left.f_{\bar{q} / Q}\right)$ are given in ref. 21] and represented by the Feynman diagrams shown in fig.2.

\section{Static properties of the nucleon}

In this section we give the basic expressions in our quark-diquark model for the magnetic moments, the isovector and isoscalar axial vector coupling constants and the pion- nucleon coupling constant. The Feynman diagrams for the valence quark contributions and the pionic cloud effects are the same as those for the LC momentum distributions (see Figs. 1 and 2) with the appropriate operator insertions, where now we have to integrate over all four components of the loop momenta. The actual evaluation of these Feynman diagrams is similar to the one described in ref. 18. 


\subsection{Magnetic moments}

For the electromagnetic current in the valence quark picture, we insert the operator $Q_{q} \gamma^{\mu}$ into the diagrams of Fig.1, where $Q_{q}$ is the charge operator for the quark. Separating the isospin matrix elements, we write for the magnetic moments of the proton and neutron:

$$
\begin{aligned}
& \mu_{p}=\frac{2}{3} \mathcal{F}_{Q}^{s}+\frac{1}{3} \mathcal{F}_{D}^{s}+\mathcal{F}_{D}^{a}+\frac{1}{\sqrt{3}} \mathcal{F}_{D}^{m} \\
& \mu_{n}=-\frac{1}{3} \mathcal{F}_{Q}^{s}+\frac{1}{3} \mathcal{F}_{D}^{s}+\frac{1}{3} \mathcal{F}_{Q}^{a}-\frac{1}{3} \mathcal{F}_{D}^{a}-\frac{1}{\sqrt{3}} \mathcal{F}_{D}^{m},
\end{aligned}
$$

where the subscripts $Q$ and $D$ refer to the quark and diquark diagrams of fig. 1 , respectively, and the superscripts $s, a$ and $m$ denote the contributions due to the scalar diquark, the a.v. diquark, and the scalar-a.v. mixing terms, respectively. These quantities are obtained by expressing the contribution to the current from the quark diagram $\left(j_{Q}^{\mu}\right)$ and the diquark diagram $\left(j_{D}^{\mu}\right)$ in terms of Dirac-Pauli form factors,

$$
\begin{aligned}
j_{R}^{\mu}\left(p^{\prime}, p\right) & =\bar{\Gamma}_{N}^{a}\left(p^{\prime}\right) \lambda_{R, a b}^{\mu}\left(p^{\prime}, p\right) \bar{\Gamma}_{N}^{b}(p) \\
& \equiv \sum_{d=s, a, m} \bar{u}_{N}\left(p^{\prime}\right)\left[\mathcal{F}_{1 R}^{d}\left(q^{2}\right) \gamma^{\mu}+\mathcal{F}_{2 R}^{d}\left(q^{2}\right) \frac{i \sigma^{\mu \nu} q_{\nu}}{2 M_{N}}\right] u_{N}(p)
\end{aligned}
$$

where $R=Q, D$. There are no mixing terms from the quark current diagram $\left(\mathcal{F}_{1 Q}^{m}=\mathcal{F}_{2 Q}^{m}=0\right.$.) Then the various contributions to the magnetic moments

in eq. (4.1) and (4.2) are obtained by $\mathcal{F}_{Q}^{d}=\mathcal{F}_{1 Q}^{d}(0)+\mathcal{F}_{2 Q}^{d}(0)(d=s, a)$ and $\mathcal{F}_{D}^{d}=\mathcal{F}_{1 D}^{d}(0)+\mathcal{F}_{2 D}^{d}(0)(d=s, a, m)$.

The vertices $\lambda_{Q, a b}^{\mu}$ and $\lambda_{D, a b}^{\mu}$ in (4.3) are obtained from Fig.1 as follows:

$$
\begin{aligned}
\lambda_{Q, a b}^{\mu}\left(p^{\prime}, p\right) & =-\int \frac{d^{4} k}{(2 \pi)^{4}} S_{F}(k+q) \gamma^{\mu} S_{F}(k) \tau_{a b}(p-k) \\
\lambda_{D, a d}^{\mu}\left(p^{\prime}, p\right) & =-i \int \frac{d^{4} k}{(2 \pi)^{4}} S_{F}(p-k) \tau_{a b}\left(k^{\prime}\right) \Lambda_{b c}^{\mu}\left(k^{\prime}, k\right) \tau_{c d}(k),
\end{aligned}
$$


where the 3-point function for the electromagnetic current of the diquark is given by

$$
\Lambda_{a b}^{\mu}\left(k^{\prime}, k\right)=3 i \int \frac{d^{4} t}{(2 \pi)^{4}} \operatorname{tr}_{D}\left[S(t+q) \gamma^{\mu} S(t) \gamma_{b} S(t-k) \gamma_{a}\right]
$$

The evaluation of the Dirac-Pauli form factors in eq. (4.4) in the covariant three-momentum cut-off scheme proceeds as follows: For each term in the loop integrals (4.4) and (4.5) which involves Lorentz tensors like $k^{\nu}$ or $k^{\mu} k^{\nu}$, a parameterization in terms of Lorentz tensors w.r.t. the external (fixed) momenta, multiplied by Lorentz invariant functions, is introduced. In this way one derives formal expressions of the Dirac-Pauli form factors in terms of these Lorentz invariant functions. For the evaluation of the magnetic moments, it is sufficient to expand these functions up to $\mathcal{O}(q)$, and the corresponding coefficients are evaluated by introducing the 3-momentum cut-off in a particular frame, i.e; the frame where $\boldsymbol{p}=0$ for the $k$-integrals in (4.4) and (4.5), while in the case of the diquark current (4.6) one chooses the frame $\boldsymbol{k}=0$ to introduce the cut-off and then generalizes the result to an arbitrary frame. (This generalization is necessary because one has to insert the general Lorentz covariant parameterization of the diquark current into (4.5) before evaluating the $k$-integral.)

The simplest case is the integral (4.4) for the scalar diquark: Besides scalar functions, the integrand involves terms $\propto k^{\nu}$ and $k^{\mu} k^{\nu}$, and these terms are most easily parameterized in terms of Lorentz tensors made of $P^{\nu} \equiv\left(p+p^{\prime}\right)^{\nu}$ and $q^{\nu}$. The most complicated case is the integral (4.5) for the a.v. diquark: Here one has to insert the following parameterization of the 3-point function (4.6) for the a.v. diquark [18]:

$$
\Lambda_{\nu \lambda}^{\mu}\left(k^{\prime}, k\right)=F_{1}(K, q) g_{\nu \lambda} K^{\mu}+F_{2}(K, q) k_{\nu}^{\prime} g_{\lambda}^{\mu}+F_{3}(K, q) k_{\lambda} g_{\nu}^{\mu}
$$




$$
\left.+F_{4}(K, q) k_{\nu}^{\prime} k_{\lambda} K^{\mu}+F_{M}(K, q)\left(g_{\lambda}{ }^{\mu} q_{\nu}-g_{\nu}{ }^{\mu} q_{\lambda}\right)\right]
$$

where $K=k^{\prime}+k$ and $q=k^{\prime}-k$, and the scalar functions depend on $K^{2}, q^{2}$ and $K \cdot q$. For $q \rightarrow 0, F_{M}$ is the "magnetic moment" of an offshell a.v. diquark 0 . This parameterization is introduced into (4.5), and a further parameterization is introduced in order to express $\lambda_{D}^{\mu}\left(p^{\prime}, p\right)$ in terms of $P^{\mu}$ and $q^{\mu}$. In this way one derives the formal expressions for the diquark contributions to the Dirac-Pauli form factors of eq. (4.4) in terms of Lorentz scalar functions, and then one can set $q=0$ for the evaluation of the magnetic moments. Similarly, for the scalar-a.v. mixing terms one has the structure

$$
\Lambda_{5 \lambda}^{\mu}\left(k^{\prime}, k\right)=F_{m}(K, q) \epsilon_{\lambda \alpha \beta}^{\mu} K^{\alpha} q^{\beta},
$$

which determines the mixing part of the vertex (4.5) and its contribution to the Dirac-Pauli form factors of the nucleon.

\subsection{Axial and pion-nucleon coupling constants}

To obtain the isoscalar $(\alpha=0)$ and isovector $(\alpha=3)$ axial vector currents in the valence quark picture, we insert the operator $\tau^{\alpha} \gamma^{\mu} \gamma_{5}$ into the diagrams of Fig.1, where $\tau^{0} \equiv 1$. The pion absorption current is obtained similarly by inserting the operator $g \tau^{3} \gamma_{5}$, where the pion- quark coupling constant $g$ is the residue of $\tau_{\pi}$ (eq.(2.5)) at the pion pole. As we will explain at the end of this subsection, in order to be consistent with the PCAC relation one

\footnotetext{
${ }^{6}$ For an on-shell a.v. diquark one naively expects [18] $F_{M}(q=0)=2$ for the same reason why the magnetic moment of the deuteron is naively expected to be the sum of the proton and neutron magnetic moments. We confirmed that for an on-shell a.v. diquark this is indeed valid almost independently of the choice of parameters. In our calculations to be discussed below, however, the a.v. diquark is always unbound. In this case the "magnetic moment" depends on the virtuality of the a.v. diquark and can assume values much less than 2 .
} 
has to take into account also the contribution from the "exchange diagram" (Fig. 3) for the pion absorption current, even in the static approximation used here. Therefore, separating again the isospin matrix elements, we write for the axial vector coupling constants $g_{A}^{(\alpha)}$ and the pion-nucleon coupling constant $g_{\pi N N}$ :

$$
\begin{aligned}
g_{A}^{(0)} & =\mathcal{G}_{Q}^{s}+\mathcal{G}_{Q}^{a}+2 \mathcal{G}_{D}^{a} \\
g_{A}^{(3)} & =\mathcal{G}_{Q}^{s}-\frac{1}{3} \mathcal{G}_{Q}^{a}+\frac{4}{3} \mathcal{G}_{D}^{a}+\frac{2}{\sqrt{3}} \mathcal{G}_{D}^{m} \\
g_{\pi N N} & =\mathcal{I}_{Q}^{s}-\frac{1}{3} \mathcal{I}_{Q}^{a}+\frac{4}{3} \mathcal{I}_{D}^{a}+\frac{2}{\sqrt{3}} \mathcal{I}_{D}^{m}-\mathcal{I}_{E}^{s}-\frac{5}{3} \mathcal{I}_{E}^{a}+\frac{1}{\sqrt{3}} \mathcal{I}_{E}^{m},
\end{aligned}
$$

where the subscript $E$ in (4.11) refers to the exchange diagram of fig.3, and the meanings of the other symbols are as in the previous subsection. The quantities $\mathcal{G}$ and $\mathcal{I}$ introduced above are obtained by expressing the axial and pion absorption currents for the quark diagram $\left(j_{A Q}^{\mu}\right.$ and $\left.j_{\pi Q}\right)$, the diquark diagram $\left(j_{A D}^{\mu}\right.$ and $\left.j_{\pi D}\right)$ and the exchange diagram $\left(j_{\pi E}\right)$ in terms of form factors as follows:

$$
\begin{aligned}
j_{A R}^{\mu}\left(p^{\prime}, p\right) & =\bar{\Gamma}_{N}^{a}\left(p^{\prime}\right) \lambda_{A R, a b}^{\mu}\left(p^{\prime}, p\right) \bar{\Gamma}_{N}^{b}(p) \\
& \equiv \sum_{d=s, a, m} \bar{u}_{N}\left(p^{\prime}\right)\left[\mathcal{G}_{R}^{d}\left(q^{2}\right) \gamma^{\mu} \gamma_{5}+\mathcal{H}_{R}^{d}\left(q^{2}\right) q^{\mu} \gamma_{5}\right] u_{N}(p) \\
j_{\pi R}\left(p^{\prime}, p\right) & =\bar{\Gamma}_{N}^{a}\left(p^{\prime}\right) \lambda_{\pi R, a b}\left(p^{\prime}, p\right) \bar{\Gamma}_{N}^{b}(p) \\
& \equiv \sum_{d=s, a, m} \bar{u}_{N}\left(p^{\prime}\right)\left[\mathcal{I}_{R}^{d}\left(q^{2}\right) \gamma_{5}\right] u_{N}(p)
\end{aligned}
$$

where $R=Q, D$ in eq. (4.13) and $R=Q, D, E$ in (4.15). The quantities $\mathcal{G}$ and $\mathcal{I}$ in eqs. (4.9)-(4.11) are then obtained by setting $q^{2}=0$ in the corresponding form factors defined in (4.13) and (4.15).

The axial vertices $\lambda_{A Q}^{\mu}$ and $\lambda_{A D}^{\mu}$ are obtained from the corresponding expressions (4.4) and (4.6) for the electromagnetic case by the replacement 
$\gamma^{\mu} \rightarrow \gamma^{\mu} \gamma_{5}$, while the pionic vertices $\lambda_{\pi Q}$ and $\lambda_{\pi D}$ are obtained by $\gamma^{\mu} \rightarrow g \gamma_{5}$. The 3-point functions corresponding to (4.6), $\Lambda_{A}^{\mu}$ and $\Lambda_{\pi}$, have the following Lorentz structures:

$$
\begin{aligned}
\Lambda_{A, \nu \lambda}^{\mu}\left(k^{\prime}, k\right) & =\epsilon^{\mu}{ }_{\nu \lambda \sigma} G(K, q) K^{\sigma} \\
\Lambda_{A, 5 \lambda}^{\mu}\left(k^{\prime}, k\right) & =g^{\mu}{ }_{\lambda} G_{m 1}(K, q)+K^{\mu} K_{\lambda} G_{m 2}(K, q) \\
\Lambda_{\pi, \nu \lambda}\left(k^{\prime}, k\right) & =\epsilon^{\alpha \beta}{ }_{\nu \lambda} I(K, q) K_{\alpha} q_{\beta} \\
\Lambda_{\pi, 5 \lambda}\left(k^{\prime}, k\right) & =I_{m 1}(K, q) K_{\lambda}+I_{m 2}(K, q) q_{\lambda},
\end{aligned}
$$

where for the axial vertices (4.16), (4.17) we show only the terms relevant in the limit $q \rightarrow 0$.

Before giving the expression for the contribution of the exchange diagram $\left(\lambda_{\pi E}\right)$ to the pionic vertex (4.15), we explain why it is necessary to consider this diagram even in the static approximation: Let us first refer to the case of the electromagnetic interaction. The Ward-Takahashi identity for the quarkphoton vertex $\Gamma^{\mu}$ is

$$
S\left(k^{\prime}\right) q_{\mu} \Gamma^{\mu} S(k)=Q_{q}\left(S(k)-S\left(k^{\prime}\right)\right) .
$$

Applying this identity to the quark-photon vertex in the exchange diagram (the cross in fig.3), we see that in the static limit for the exchanged quark $(S(k) \rightarrow-1 / M)$, the r.h.s. of this identity is zero. It is therefore consistent to assume that in the static approximation there is no contribution from the exchange diagram, as has been done in previous works [25]. 7 If there were no violations of gauge invariance due to the cut-off procedure, the electromagnetic current of the nucleon calculated from the quark and diquark diagrams

\footnotetext{
${ }^{7}$ The same argument holds also for the baryon current, and therefore no contributions from the exchange diagram are needed to satisfy the quark number sum rules in the static approximation.
} 
alone would satisfy the current conservation $q_{\mu} J^{\mu}=0$. Similarly, if we apply the axial Ward-Takahashi identity

$$
S\left(k^{\prime}\right) q_{\mu} \boldsymbol{\Gamma}_{A}^{\mu} S(k)=\boldsymbol{\tau}\left(\gamma_{5} S(k)+S\left(k^{\prime}\right) \gamma_{5}\right)+2 M S\left(k^{\prime}\right) \gamma_{5} \boldsymbol{\tau} S(k)
$$

to the quark axial vertex in the exchange diagram of fig.3, we note that in the static limit $(S(k) \rightarrow-1 / M)$ the r.h.s. of (4.21) vanishes, too, which suggests that the exchange diagram should not be taken into account in the calculation of the axial vector current in the static approximation. If there were no violations of chiral invariance of different origin 8 , the axial current of the nucleon calculated from the quark and diquark diagrams alone would satisfy the PCAC relation (with the pion pole contributions subtracted): $q_{\mu} J_{A}^{\mu}(q)=f_{\pi} J_{\pi}(q)$, where $f_{\pi}=M / g$. The pion absorption current $J_{\pi}$ in this relation, however, includes the effects of the exchange diagram, since in our above discussion the last term in eq. (4.21) just corresponds to the contribution of the exchange diagram to $J_{\pi}$. In other words, if $g_{A}^{(3)}$ is calculated from the quark and diquark diagrams alone, the corresponding pion-nucleon coupling constant in the Goldberger-Treiman relation $M_{N} g_{A}^{(3)}=g_{\pi N N} f_{\pi}$ includes the effect of the exchange diagram. In Appendix B we explicitly show the validity of the PCAC relation for the case where only the scalar diquark channel is included, both in the exact Faddeev framework and in the static approximation.

\footnotetext{
${ }^{8}$ In the present calculation, however, there are 2 origins of violation of PCAC and the Goldberger- Treiman relation: The first reason is the omission of the vector diquark channel, as has been explained in sect. 2. The second, and probably more important, reason is that the 3-momentum (or Lepage-Brodsky) cut-off scheme used here satisfies charge and baryon number conservation, but not the full vector current conservation and PCAC. Since in the static approximation used in this work the quark- diquark vertex function is independent of the relative momentum, the dependence of the quark-diquark loop integrals on the cut-off is enhanced considerably, and this leads to sizeable violations of the Goldberger-Treiman relation as will be discussed in sect. 5 .
} 
The contribution of the exchange diagram to the pionic vertex of eq. (4.14) in the static approximation can be expressed in terms of the quarkdiquark bubble graph $\Pi_{N}$ of eq. (2.11) by

$$
\lambda_{\pi E}^{a b}=-3 g \Pi_{N}^{a c}\left(p^{\prime}\right) \gamma_{c}\left(\frac{1}{M^{2}} \gamma_{5}\right) \gamma_{d} \Pi_{N}^{d b}(p),
$$

which includes a color factor 3 . If only the scalar diquark channel is included, the Faddeev kernel in the static approximation is equal to $\Pi_{N}(p) \cdot 3 / M$ (see (2.9)), which simplifies the calculation of the exchange diagram contribution to the pion absorption current (4.15).

The explicit evaluation of the various contributions shown in eqs. (4.9)(4.11) in the covariant three-momentum cut-off scheme proceeds along the lines discussed in the previous subsection.

\subsection{Pion cloud effects}

Although the main purpose of this work is to extract information on the strength of the a.v. diquark correlations in the nucleon, we also should estimate the size of the pion cloud effects in the present quark-diquark model. The purpose of this subsection is to explain a simple way, involving various approximations, to estimate these effects. These approximations, of course, should be avoided in a more refined treatment of pion cloud effects. (For a more general recent discussion on pionic effects on the static properties of the nucleon, we refer to ref. [26].)

To estimate the role of pion cloud effects on the static properties of the nucleon, we use an on-shell approximation for the "parent quark", which has been used also to estimate the pion cloud effects on the structure functions (see ref. [?]). That is, the operator insertions on the quark lines in fig. 1 
are replaced by the diagrams of fig. 2, but these diagrams are evaluated by assuming that the external quark lines are on the mass shell. For the calculation of $g_{A}^{(\alpha)}$ this simply means that we replace the 'bare' axial vector coupling constant of the quark (which is 1 ) by $g_{A q}^{(\alpha)}$, which includes the effect of the pion dressing, and similarly for $g_{\pi N N}$ we replace the bare $\pi q q$ coupling $(g)$ by the dressed one $\left(g_{\pi q q}\right)$ f?. For the calculation of magnetic moments, there appears in principle a new type of quark operator associated with the anomalous magnetic moment of the constituent quark. In a non-relativistic approximation, however, the matrix element of this operator can be related to the isoscalar and isovector axial vector coupling constants of the nucleon, see eq. (4.31) below.

The magnetic moments of the $u$ and $d$ quarks including the pion dressing are written as

$$
\begin{aligned}
& \mu_{u}=Z_{q} Q_{u}+3 \mathcal{F}_{\pi} \equiv Q_{u}+\kappa_{u} \\
& \mu_{d}=Z_{q} Q_{d}+\mathcal{F}_{q}-\mathcal{F}_{\pi} \equiv Q_{d}+\kappa_{d}
\end{aligned}
$$

The quantities $\mathcal{F}_{q}$ and $\mathcal{F}_{\pi}$ are obtained by expressing the results for the quark diagram $j_{q}^{\mu}$ (first diagram of fig. 2) and the pionic diagram $j_{\pi}^{\mu}$ (second diagram of fig. 2) in terms of Dirac-Pauli form factors:

$$
\begin{aligned}
j_{r}^{\mu}\left(p^{\prime}, p\right) & =\bar{\Gamma}_{q}\left(p^{\prime}\right) \lambda_{r}^{\mu}\left(p^{\prime}, p\right) \Gamma_{q}(p) \\
& \equiv \bar{u}_{q}\left(p^{\prime}\right)\left[\mathcal{F}_{1 r}\left(q^{2}\right) \gamma^{\mu}+\mathcal{F}_{2 r}\left(q^{2}\right) \frac{i \sigma^{\mu \nu} q_{\nu}}{2 M}\right] u_{q}(p)
\end{aligned}
$$

\footnotetext{
${ }^{9}$ In order to respect chiral symmetry, one should also include the mixing between the pion and the sigma meson in the third diagram of fig. 2, but this will not be done here in this rather schematic treatment of pion cloud effects. We also note that in principle the pion cloud effects lead to modifications of the quark and diquark propagators and the quark-diquark vertex functions. However, if the quark propagator is approximated by its pole part, these modifications can be absorbed by a redefinition of the constituent quark mass and the 4-fermi coupling constants, as has been discussed in ref. [21]
} 
where $r=q, \pi$. The contributions to the magnetic moments in eq. (4.23) and (4.24) are then obtained by $\mathcal{F}_{q}=\mathcal{F}_{1 q}(0)+\mathcal{F}_{2 q}(0)$ and $\mathcal{F}_{\pi}=\mathcal{F}_{1 \pi}(0)+\mathcal{F}_{2 \pi}(0)$. The quark spinor $\Gamma_{q}(p)$ in (4.25) is given by $\Gamma_{q}(p)=\sqrt{Z_{q}} u_{q}(p)$, where the free Dirac spinor $u_{q}(p)$ involves the quark mass $M$, and the normalization factor, which also appears explicitly in (4.23) and (4.24), is given by 21

$$
Z_{q}=\left(1+\left.\frac{\partial \prod_{q}(k)}{\partial \not k}\right|_{\not k=M}\right)^{-1}
$$

with the quark self energy

$$
\Pi_{q}(k)=3 \int \frac{d^{4} q}{(2 \pi)^{4}}\left[\gamma_{5} S_{F}(q) \gamma_{5}\right] \tilde{\tau}_{\pi}(k-q) .
$$

The vertices $\lambda_{q}^{\mu}$ and $\lambda_{\pi}^{\mu}$ in (4.25) are obtained from the diagrams of Fig.2 as follows:

$$
\begin{aligned}
& \lambda_{q}^{\mu}\left(p^{\prime}, p\right)=-\int \frac{d^{4} k}{(2 \pi)^{4}} S_{F}(k+q) \gamma^{\mu} S_{F}(k) \tilde{\tau}_{\pi}(p-k) \\
& \lambda_{\pi}^{\mu}\left(p^{\prime}, p\right)=-i \int \frac{d^{4} k}{(2 \pi)^{4}} S_{F}(p-k) \tau_{\pi}\left(k^{\prime}\right) \Lambda_{\pi}^{\mu}\left(k^{\prime}, k\right) \tau_{\pi}(k),
\end{aligned}
$$

where the 3-point function for the electromagnetic current of the pion is given by

$$
\Lambda_{\pi}^{\mu}\left(k^{\prime}, k\right)=3 i \int \frac{d^{4} t}{(2 \pi)^{4}} \operatorname{tr}_{D}\left[S(t+q) \gamma^{\mu} S(t) \gamma_{5} S(t-k) \gamma_{5}\right] .
$$

The reduced pion t-matrix $\tilde{\tau}_{\pi}$ in the above expressions is defined so as to exclude the contribution of the 'bare' contact interaction एण: $\tilde{\tau}_{\pi} \equiv \tau_{\pi}+2 i G_{\pi}$.

\footnotetext{
${ }^{10}$ The contribution of the contact term to the vertex (4.28) is equivalent to an "RPAtype" vertex correction induced by an interaction of vector type $\left(\propto\left(\bar{\psi} \gamma^{\mu} \tau^{\alpha} \psi\right)^{2}\right)$ with a strength determined by the Fierz transformation of the pseudoscalar interaction term in the lagrangian. However, our strategy is to consider our underlying interaction Lagrangian in the $q \bar{q}$ channels to be already Fierz symmetrized, and to discard all channels except the scalar-pseudoscalar one in order not to introduce additional parameters (see sect. 2).
} 
Having determined the magnetic moments (4.23), (4.24) of the on-shell parent quark in the way described above, one should then use the quark operator $\frac{i \sigma^{\mu \nu} q_{\nu}}{2 M} \kappa_{q}$, where $\kappa_{q}=\frac{1}{2}\left(1+\tau_{3}\right) \kappa_{u}+\frac{1}{2}\left(1-\tau_{3}\right) \kappa_{d} \equiv \kappa_{q}^{(0)}+\tau_{3} \kappa_{q}^{(3)}$, to calculate the contribution to the nucleon magnetic moments as described in the previous subsection. In a non-relativistic approximation for the constituent quark, however, this operator (for $\mu=i$ ) can be replaced by $\frac{i}{2 M}\left(\boldsymbol{\gamma} \gamma_{5}\right) \times \boldsymbol{q} \kappa_{q}$, and then the contribution of the pion cloud to the anomalous magnetic moment of the nucleon can be written as

$$
\kappa_{p(n)}^{(\pi)}=\frac{M_{N}}{M}\left(g_{A}^{(0)} \kappa_{q}^{(0)} \pm g_{A}^{(3)} \kappa_{q}^{(3)}\right)
$$

with + and - for proton and neutron, respectively.

Similarly, the axial vector and $\pi q q$ coupling constants of the on- shell parent quark including the pion dressing are written as

$$
\begin{aligned}
g_{A q}^{(0)} & =Z_{q}+3 \mathcal{G}_{q} \\
g_{A q}^{(3)} & =Z_{q}-\mathcal{G}_{q} \\
g_{\pi q q} & =Z_{q} g-\mathcal{I}_{q} .
\end{aligned}
$$

In this case, there is no contribution due to the pion current (the third diagram in fig. 2). These quantities $\mathcal{G}_{q}$ and $\mathcal{I}_{q}$ are obtained by expressing the axial and pion absorption currents for the quark diagram $\left(j_{A q}^{\mu}\right.$ and $\left.j_{\pi q}\right)$ in terms of form factors as follows:

$$
\begin{aligned}
j_{A q}^{\mu}\left(p^{\prime}, p\right) & =\bar{\Gamma}_{q}\left(p^{\prime}\right) \lambda_{A q}^{\mu}\left(p^{\prime}, p\right) \bar{\Gamma}_{q}(p) \\
& \equiv \bar{u}_{q}\left(p^{\prime}\right)\left[\mathcal{G}_{q}\left(q^{2}\right) \gamma^{\mu} \gamma_{5}+\mathcal{H}_{q}\left(q^{2}\right) q^{\mu} \gamma_{5}\right] u_{q}(p) \\
j_{\pi q}\left(p^{\prime}, p\right) & =\bar{\Gamma}_{q}\left(p^{\prime}\right) \lambda_{\pi q}\left(p^{\prime}, p\right) \bar{\Gamma}_{q}(p) \\
& \equiv \bar{u}_{q}\left(p^{\prime}\right)\left[\mathcal{I}_{q}\left(q^{2}\right) \gamma_{5}\right] u_{q}(p),
\end{aligned}
$$


The quantities $\mathcal{G}_{q}$ and $\mathcal{I}_{q}$ in eqs. (4.32)-(4.34) are then obtained by setting $q^{2}=0$ in the form factors defined in (4.36) and (4.38). The axial vertex $\lambda_{A q}^{\mu}$ in (4.35) is obtained from the corresponding expression (4.28) for the electromagnetic case by the replacement $\gamma^{\mu} \rightarrow \gamma^{\mu} \gamma_{5}$, while the pionic vertex $\lambda_{\pi q}$ in (4.37) is obtained by replacing $\gamma^{\mu} \rightarrow g \gamma_{5}$. The isoscalar and isovector axial vector coupling constants of the nucleon are then renormalized simply by multiplying the factors $g_{A q}^{(0)}$ and $g_{A q}^{(3)}$, respectively, while the $\pi N N$ coupling constant gets multiplied by $g_{\pi q q} / g$.

\section{Results and discussions}

In this section we will discuss our results for the nucleon structure functions and static properties. First we have to explain the choice of our parameters. The three basic parameters of the NJL model, namely the 4-fermi coupling constant in the pionic channel $G_{\pi}$, the UV cut-off $\Lambda$, and the current quark mass $m$, are determined so as to reproduce the pion mass $m_{\pi}=140 \mathrm{MeV}$ as the pole of $\tau_{\pi}$ (eq.(2.5)), the pion decay constant $f_{\pi}=93 \mathrm{MeV}$ via the familiar quark loop diagram for charged pion decay [12], and the constituent quark mass $M=400 \mathrm{MeV}$ via the gap equation. (The qualitative behaviour of the results does not depend on this particular choice for $M$.) The resulting parameters in the 3 -momentum cut-off scheme are $G_{\pi}=6.92 \mathrm{GeV}^{-2}, \Lambda=$ $593 \mathrm{MeV}$, and $m=5.96 \mathrm{MeV}$.

In order to see the dependence of our results on the strength of the a.v. diquark correlations, we consider the ratio $r_{a}=G_{a} / G_{\pi}$ as a free parameter, and adjust the strength in the scalar diquark channel $r_{s}=G_{s} / G_{\pi}$ so as to reproduce the experimental nucleon mass $M_{N}=940 \mathrm{MeV}$ from the Faddeev 
equation in the static approximation (eq.(2.9)). Table 1 shows 3 particular choices for $r_{a}$. Case I refers to the pure scalar diquark model of ref. [21], which leads to a strongly bound scalar diquark of mass $M_{s}=596 \mathrm{MeV}$. For case III the value $r_{a}=0.66$ is strong enough to produce a bound delta state of mass $M_{\Delta}=1140 \mathrm{MeV}$, but nevertheless the a.v. diquark is still unbound. The corresponding value of $r_{s}$ is much smaller than that in case I to give the same $M_{N}$. This case therefore refers to weak scalar diquark correlations with a weakly bound scalar diquark of mass $M_{s}=766 \mathrm{MeV}$. Case II $\left(r_{a}=0.25\right)$ describes a situation intermediate between these two. For each case we also show in Table 1 the corresponding "weight" $W_{s}$ of the scalar diquark channel, which is defined as the contribution to the baryon number (see eq.(2.16)).

In order to compare the calculated structure functions and quark LC momentum distributions with the empirical ones, we have to associate a "low energy scale" $\mu^{2} \equiv Q_{0}^{2}$ to our NJL model results, and perform the $Q^{2}$ evolution up to the value of $\mu^{2}=Q^{2}$ where experimental data and empirical parameterizations are available. For this purpose we solve the DGLAP equation [27] in the $\overline{\mathrm{MS}}$ scheme up to next-to-leading order, using the computer code of ref. [28] with $N_{f}=3$ and $\Lambda_{Q C D}=250 \mathrm{MeV}$, and compare with empirical parameterizations which also refer to the $\overline{\mathrm{MS}}$ scheme. If we determine $Q_{0}^{2}$ so as to reproduce the overall features of the empirical valence quark distributions (see fig. 7 below), we obtain $Q_{0}^{2}=0.16 \mathrm{GeV}^{2}$, i.e., $Q_{0}$ is equal to our constituent quark mass $M$.

\subsection{Structure functions}

Since the main purpose of this work is to investigate the role of a.v. diquark correlations on the flavor dependence of the structure functions, we 
discuss this point first. In Fig. 4 we compare the valence quark distributions (multiplied by $x$ ) $\frac{1}{2} x u_{v}(x)$ and $x d_{v}(x)$, without pion cloud effects, for the three cases I, II and III of Table 1. (The factor $\frac{1}{2}$ is multiplied in order to compare distributions with the same normalization.) Case I shows a rather large 'flavor asymmetry', i.e., $d_{v}$ is softer than $u_{v}$. This is a typical example for strong scalar diquark correlations, as has been discussed first in ref. [0]: If $M_{s}$ is small, the scalar diquark carries a LC momentum fraction which is small compared to the case of a weakly bound state $\left(M_{s} \simeq 2 M\right)$, and accordingly the spectator quark carries a large fraction of the LC momentum. Since the scalar diquark consists of a $u d$ pair, this implies that for strong scalar diquark correlations the $u$ quark distribution in the proton has a 'hard' component, while the $d$ quark distribution has only a 'soft' component. In terms of the distributions given by (3.2) and (3.3), the hard component is the term $F_{Q / P}^{s}(x)$ due to the quark diagram, while the soft component is $F_{Q(D) / P}^{s}(x)$ due to the diquark diagram. For case I, the two valence $u$ quarks in the proton carry $71 \%$ of the LC momentum, while the valence $d$ quark carries only $29 \%$. This flavor asymmetry is gradually decreased as we increase $r_{a}$, as is shown in Fig.4. For the cases II (III), the two valence $u$ quarks carry 69 $\%(68 \%)$ of the total LC momentum. Particularly important is the fact that for large $x$ the valence $d$ quark distribution increases rapidly with increasing $r_{a}$ and approaches the valence $u$ quark distribution.

The influence of the a.v. diquark correlations on the flavor asymmetry of the valence quark distributions is mainly an indirect one: If one increases $r_{a}$ one has to choose a smaller $r_{s}$ in order to get the same nucleon mass, which implies that $M_{s}\left(W_{s}\right)$ increases (decreases) with increasing $r_{a}$. The direct influence of the a.v. diquark channel on the flavour asymmetry is small, since 
even for case III the a.v. diquark correlations are 'small' compared to the scalar ones in the sense that the a.v. diquark is still unbound. In terms of the distributions (3.2) and (3.3), this means that $F_{Q(D) / P}^{a}(x) \simeq 2 F_{Q / P}^{a}(x)$. One should also note that, due to the same reason, the a.v. diquark correlations lead to a larger width of the valence quark distributions: Since $M_{s}$ increases as we increase the ratio $r_{a}$, the binding energy of the diquark-quark system increases because the nucleon mass is fixed, and as a consequence the width of the valence quark distributions increases.

Let us now discuss the flavor dependence of our valence quark distributions in connection with the empirical information. The quantity which is most sensitive to the flavor asymmetry of the valence quark distributions is the ratio of the neutron to the proton structure function $R(x)=F_{2}^{n}(x) / F_{2}^{p}(x)$ for large $x$ [8]. Flavor symmetric distributions $\left(d_{v}(x) / u_{v}(x)=\frac{1}{2}\right)$, like in the naive $\mathrm{SU}(6)$ quark model, give a constant value $R(x)=2 / 3$, while for the case of a strong dominance of scalar diquark correlations over the a.v. ones one expects $d_{v}(x) / u_{v}(x) \rightarrow 0$, or $R \rightarrow 1 / 4$, as $x \rightarrow 1$. Our calculated ratios $R(x)$ for $Q^{2}=12 \mathrm{GeV}^{2}$ are plotted for several values of $r_{a}$ in Fig. 5. The influence of the a.v. diquark correlations on the flavor dependence of the valence quark distributions is clearly seen: For large $x$ the ratio gradually increases with increasing $r_{a}$ (decreasing strength of the scalar diquark correlations). To compare this behaviour with the experimental data, one must note that $F_{2}^{n}$ has to be extracted from the experimental deuteron structure function [2], and therefore some model dependence is introduced. A very careful re-analysis has been performed in ref.[B], including binding and offshell effects. The lower data points shown in Fig. 5 have been extracted from the SLAC proton and deuteron data [2] by using a deuteron model with on- 
shell nucleons, while the upper data points are taken from the re-analysis of ref. [8]. From Fig. 5 we see that the empirical data, in particular the re-analyzed ones, seem to require some admixture of a.v. diquark components. The comparison with the re-analyzed data favors values for $r_{a}$ which are not too far from case II $\left(r_{a}=0.25\right)$ of Table 1 . Case III, which is characterized by strong a.v. diquark correlations with a bound delta state, is in clear contradiction with the data. 円 Later we will investigate whether values around $r_{a} \simeq 0.25$, corresponding to a dominant scalar diquark component with weight $\simeq 93 \%$, are favored also by other observables of the nucleon.

In fig. 6 we show the valence quark distributions for case II $\left(r_{a}=0.25\right)$ of Table 1, including pion cloud effects. The distributions calculated in the NJL model (solid lines) are evolved up to $Q^{2}=4 \mathrm{GeV}^{2}$ (dashed lines) and compared to the empirical distributions extracted in a recent analysis from the experimental data (dotted lines) 世2. Compared to the results of the pure scalar diquark model (case I of Table 1) presented in ref. 21], we note that the a.v. diquark effects work towards a better agreement with the empirical distributions, mainly due to the increase of the width of the distributions.

The antiquark distributions shown in Fig. 7 for the case II are not sen-

\footnotetext{
${ }^{11}$ As discussed in ref. [8], QCD calculations based on hard gluon exchange [29] show that $d_{v}(x) / u_{v}(x) \rightarrow \frac{1}{5}(R(x) \rightarrow 3 / 7)$ as $x \rightarrow 1$, in agreement with quark counting rules [30]. In our NJL model calculation, the ratio $R(x)$ for large $x$ is determined mainly by the strength of the a.v. diquark correlations. One should note, however, that there is no fundamental relation between the a.v. diquark correlations discussed here and the hard gluon exchange correlations discussed in 29. For example, the $x \rightarrow 1$ behaviour of our distributions depends to some extent on the cut-off scheme (although we did not investigate whether also the ratio is sensitive to the cut-off scheme), and the lines in Fig.5 simply reflect the change of the probability of the $S=1$ quark pair without any preference of $S_{z}=0$ pairs, in contrast to the hard gluon exchange model.

${ }^{12}$ The pion cloud effects taken into account in this work make the valence quark distributions softer, i.e., reduce their peak heights and increase the support at low $x$, but do not give rise to any substantial flavor dependence.
} 
sitive to the a.v. diquark correlations. As in the pure scalar diquark model of ref. 21], we see that the pion cloud effects taken into account in this work do lead to an enhancement of $\bar{d}$ over $\bar{u}$, but this flavor asymmetry of the Dirac sea is too small for intermediate values of $x$. The calculated value of the Gottfried sum $S_{G}=0.262$ agrees well with the experimental value $(0.235 \pm 0.026)$ [1], since at very low $x$ our calculated distributions show a rather large asymmetry. For the case II shown here, $92 \%$ of the total LC momentum is carried by the valence quarks at the low energy scale, and the rest is carried by the sea quarks.

Fig. 8 shows the proton structure function $F_{2 p}(x)$ calculated for the case II at $Q^{2}=4.5 \mathrm{GeV}^{2}$ and $Q^{2}=15 \mathrm{GeV}^{2}$ including pion cloud effects, in comparison with the experimental data of ref. [3]. It is seen that the present quark- diquark model calculation reproduces the overall features of the experimental structure function.

\section{$5.2 \quad$ Static properties}

We now turn to the dependence of the results for the static properties of the nucleon on the a.v. diquark correlations. The proton and neutron magnetic moments are shown in Fig.9 as functions of the ratio $r_{a}$. In the pure scalar diquark model $\left(r_{a}=0\right)$, the magnetic moments are too small in magnitude, even if the pion cloud effects are taken into account. The contributions due to the a.v. diquark channel enhance the magnitudes of the magnetic moments, and for $r_{a} \simeq 0.2$ the experimental values can reproduced. Since the neutron magnetic moment is more sensitive to $r_{a}$ than the proton one, the ratio $\left|\mu_{p} / \mu_{n}\right|$ decreases with increasing $r_{a}$. Therefore, for large $r_{a}$, as would be required by a bound delta state (case III of Table 1), not only the magnitude 
of the magnetic moments but also their ratio becomes unreasonable.

For the three values of $r_{a}$ corresponding to the cases I, II and III of Table 1 , these results are split into the various contributions in Table 2. Most important is the quark diagram in the scalar channel (the term $\mathcal{F}_{Q}^{s}$ in (4.1) and (4.2) ), which increases as one goes from case I to case II, in spite of the reduced probability $W_{s}$ of the scalar diquark channel. For $\mu_{p}$, the diquark diagram in the a.v. channel $\left(\mathcal{F}_{D}^{a}\right)$ and the mixing term $\left(\mathcal{F}_{D}^{m}\right)$ give further enhancements, in particular for large values of $r_{a}$. The enhancement of $\left|\mu_{n}\right|$ is dominated by the quark diagram in the a.v. channel $\left(\mathcal{F}_{Q}^{a}\right)$, which is absent for $\mu_{p}$, and therefore the neutron magnetic moment is more sensitive to the a.v. diquark correlations. The pion cloud gives rise to the following anomalous magnetic moments of the constituent quarks (see eq. (4.23), (4.24)): $\kappa_{u}=0.26, \kappa_{d}=-0.29$. Due to eq. (4.31), the pionic contributions to the nucleon magnetic moments are mainly of isovector type, too, and enhance their magnitudes, as is well known [26].

The results for the axial vector coupling constants are shown in Fig. 10 as functions of the ratio $r_{a}$. The fact that the calculated $g_{A}^{(3)}$ is too small compared to the experimental value is most probably due to the static approximation to the Faddeev equation used in the present calculation $[3$. The a.v. diquark channel gives rise to a rather slow increase of $g_{A}^{(3)}$ and a rapid decrease of $g_{A}^{(0)}$. Since the increase of $g_{A}^{(3)}$ is slow and there is no sizeable enhancement beyond $r_{a} \simeq 0.3$, one may conclude that a.v. diquark corre-

\footnotetext{
${ }^{13}$ In the exact Faddeev calculation [16], the quark diagram in the scalar channel $\left(\mathcal{G}_{Q}^{s}\right.$ in eq. (4.10) gives a larger contribution, and also the exchange diagram gives some enhancement. In this connection one should also note that the charge radii of the nucleons are too small in the static approximation [25], indicating that relativistic effects are enhanced. This is one of the reasons why the axial vector coupling constants are smaller than in the exact Faddeev calculation.
} 
lations work in the desired direction, but there is no necessity to introduce strong correlations as would be required by a bound delta state (case III of Table 1). Similarly, the largest part of the reduction of $g_{A}^{(0)}$ occurs for small $r_{a}$, whereas for larger $r_{a}$ the curve saturates.

Table 2 shows the various contributions to the axial coupling constants for the cases I, II and III. The most sizeable effects due to the a.v. diquark channel are the positive mixing term $\left(\mathcal{G}_{D}^{m}\right.$ in eq. 4.10$)$ ) for $g_{A}^{(3)}$, and the reduction of the scalar channel contribution $\left(\mathcal{G}_{Q}^{s}\right)$. The net effect is the rather slow increase of $g_{A}^{(3)}$ and the rapid decrease of $g_{A}^{(0)}$ discussed above. The pion cloud renormalizes the axial vector coupling constants of the constituent quark (see (4.32) and (4.33)): $g_{A q}^{(3)}=0.86, g_{A q}^{(0)}=0.78$. These reductions due to the pion cloud are well known, see e.g., ref. [31]. There it was pointed out that, due to the p-wave coupling of the pion to the quarks, some amount of the nucleon spin carried by the quark spin is transfered to orbital angular momentum of the pion cloud, that is, to orbital angular momentum of the sea quarks, leading to the reduction of $g_{A q}^{(0)}$.

In Fig. 11a we show the dependence of $g_{\pi N N}$ on the ratio $r_{a}$. In this case, again, the result of the pure scalar diquark model is too small in comparison with the experimental value, and the a.v. diquark channel gives rise to a sizeable enhancement, in particular in the region of small $r_{a}$ where the experimental value can be reproduced for $r_{a} \simeq 0.3$. Similarly to the cases of $g_{A}^{(3)}$ and $\mu_{p}$, the behaviour of the curves in Fig. 11a indicates that most of the a.v. diquark correlation effects are exhausted already for admixtures which are small compared to the case III of Table 1 (that is, the case of a bound delta state). Pion cloud effects renormalize the $\pi q q$ coupling constant (see eq. (4.34)) from the bare value $g=4.23$ to $g_{\pi q q}=3.48$. Among the various 
contributions shown in Table 2, the quark diagram in the scalar channel gives the dominant contribution, followed by the diquark diagram in the a.v. channel and the exchange diagrams. There are large cancellations among the exchange diagram contributions, leaving a net positive correction.

Compared to the case of $g_{A}^{(3)}$, the $\pi N N$ coupling constant shows a rather rapid increase in the small $r_{a}$ region, which indicates a violation of the Goldberger-Treiman (GT) relation. In Fig. $11 \mathrm{~b}$ we plot the ratio $\Delta_{G T} \equiv$ $f_{\pi} g_{\pi N N} / M_{N} g_{A}^{(3)}$ as a function of $r_{a}$ (upper pair of lines). This figure shows that already for the pure scalar diquark model there is a sizeable violation of the GT relation of about 13\% (for the case including the pion cloud effects), and if the a.v. diquark channel is included this violation increases up to $90 \%$. This fact indicates a major problem of the static approximation to the Faddeev equation: In this approximation the quark-diquark vertex function is independent of the relative momentum in the quark-diquark system, and therefore all contributions to the loop integrals which are not sufficiently damped due to the internal quark or diquark propagators are very sensitive to the cut-off 14 . The terms with the highest degree of divergence are those due to the contact terms $4 i G_{s}$ and $4 i G_{a} g^{\mu \nu}$ in the scalar and a.v. t- matrices in (2.5) and (2.6), respectively. In order to demonstrate that these terms are largely responsible for the violation of PCAC, we show by the lower pair of lines in Fig. 11b the result obtained by leaving out these contact terms. (Since the contact terms act attractively, we have to choose larger values of $r_{s}$ for each $r_{a}$ in order to keep the nucleon mass fixed.) In such a calculation, where the pole term due to the scalar t-matrix gives the dominant

\footnotetext{
${ }^{14}$ As was discussed in the previous secions, the three-momentum cut-off procedure used here violates the GT relation, although it conserves baryon number, electric charge and momentum.
} 
contribution besides small corrections due to the $q q$ continuum terms, the violations of the GT relation are drastically reduced. This indicates that the large violations of the GT relation will disappear as soon as one uses the exact Faddeev wave functions.

The main purpose of this subsection was to see whether our conclusions on the strength of the a.v. diquark correlations, derived in sect. 5.1 from the flavor dependence of the quark LC momentum distributions, are consistent with the static properties of the nucleon or not. From our above discussions we can now conclude that the static properties, too, indicate the necessity of some amount of a.v. diquark correlations, but not strong ones. The range $0.15<r_{a}<0.3$, corresponding to $0.98>W_{s}>0.90$, seems reasonable from both points of view.

\section{$6 \quad$ Summary and conclusions}

The purpose of this paper was to extract information on the strength of the quark-quark interaction in the axial vector (a.v.) diquark channel by comparing the results for the structure functions and the static properties of the nucleon with the empirical information. For this purpose we used the Nambu-Jona-Lasinio (NJL) model in the framework of a simple quarkdiquark approximation ("static approximation") to the full Faddeev equation. The effects of the pion cloud were taken into account by assuming an on-shell approximation for the parent quark, which in the case of the quark distributions leads to the usual one-dimensional convolution formalism. Our results are summarized as follows:

First, the observed flavor dependence of the structure functions implies 
that the interaction in the a.v. diquark channel should be relatively weak compared to that in the scalar diquark channel. In the model used here we found that, in order to reproduce the flavor dependence, the weight of the a.v. diquark component in the nucleon state (defined here as the contribution to the baryon number) should not be much larger than $10 \%$. Since in this case a large part of the binding energy has to come from the correlations in the scalar diquark channel, this implies rather strong scalar diquark correlations in the nucleon.

Second, the static properties of the nucleon indicate that some amount of a.v. diquark correlations are required, in particular for the magnetic moments which are too small in magnitude in a pure scalar diquark model. The a.v. diquark channel gives beneficial contributions to all static properties considered here, but it is neither necessary nor preferable to introduce strong a.v. correlations. That is, a large part of the effects due to the a.v. channel on the static properties is exhausted already for an admixture of less than $10 \%$.

Combining these two observations, we conclude that an admixture of the a.v. channel between $2 \%$ and $10 \%$ seems very reasonable. In terms of the 4-fermi coupling constants, this corresponds to $r_{s} \simeq 0.63$ and $r_{a} \simeq 0.25$ (see case II of Table 1). It is interesting to note that these values are similar to $r_{s}=0.5$ and $r_{a}=0.25$, which correspond to the 'color current' type interaction lagrangian [20]. This relatively small value of $r_{a}$ implies rather weak a.v. diquark correlations, since for example the a.v. diquark is unbound and no bound state for the delta isobar can be obtained. While this latter fact at first sight seems to indicate a problem of the model, we should note that for reasonable values of the constituent quark mass the delta isobar always 
emerges very close to the (unphysical) 3-quark threshold in a model without confinement. (A similar situation holds also for the vector mesons if they are described as $q \bar{q}$ bound states.) Our results support the viewpoint that one should not attempt to describe these heavier hadrons as bound states in a model without confinement. To describe these states, one should at least incorporate one important aspect of the confinement, namely the absence of unphysical thresholds for the decay into colored states $117,32,33 \mathrm{H}$.

The numerical results obtained for an admixture between $2 \%$ and $10 \%$ of the a.v. channel show that the overall picture which emerges for the structure functions and the static properties is quite reasonable. There are, however, several points which should be improved, in particular concerning the violation of the Goldberger-Treiman relation, the shape of $d_{v}(x)$, the difference $\bar{d}(x)-\bar{u}(x)$, etc. For these purposes, further work should be done towards a full Faddeev description and a more refined treatment of pion cloud effects.

\section{ACKNOWLEDGEMENTS}

This work was supported by the Grant in Aid for Scientific Research of the Japanese Ministry of Education, Culture, Sports, Science, and Technology, Project No. C2-13640298, and Project No. C2-11640257. The authors thank M. Miyama and S. Kumano for providing them with the computer code of ref. 28] for the $Q^{2}$ evolution, W. Melnitchouk for the data points shown in Fig.

\footnotetext{
${ }^{15}$ It is interesting to note that recently a similar conclusion has been derived for the description of nuclear matter [34]: In the NJL model without confinement effects, a nucleon in the medium becomes a loosely bound state near the threshold, and the resulting equation of state does not saturate. If the thresholds are avoided, for example by introducing a proper time infra-red cut-off [33], the equation of state saturates.
} 
5 (Fig. 3 of ref. [8]), and A.W. Thomas for helpful discussions on structure functions and pion cloud effects. 


\section{References}

[1] SMC Collaboration, B. Adevara, et al., Phys. Rev. D 58 (1998) 112001.

NM collaboration, M. Arneodo et al., Phys. Rev. D 50 (1994) R1;

H1 collaboration, C. Adloff et al., Z. Phys. C 74 (1997) 191

ZEUS collaboration, J. Breitweg et al., Z. Phys. C 74 (1997) 207.

[2] L.W. Whitlow et al., Phys. Lett. B 282(1992) 475;

J. Gomez et al., Phys. Rev. D 49 (1994) 4348.

[3] NM collaboration, M. Arneodo et al., Nucl. Phys. B 483 (1997) 3.

[4] R.K. Ellis, W.J. Stirling and B.R. Webber, QCD and Collider Physics, Cambridge Univ. Press, 1996;

A. W. Thomas and W. Weise, The Structure of the Nucleon, WileyVCH, 2001.

[5] J.C. Collins, D. Soper and G. Sterman, Perturbative Chromodynamics, ed. A.H. Mueller, World Scientific, Singapore, 1989, p.1.

[6] A.D. Martin, R.G. Roberts, W.J. Stirling and R.S. Thone, Eur. Phys. J. C 14 (2000) 133.

H.L. Lai et al. [CTEQ Collaboration], Eur. Phys. J. C 12 (2000) 375.

M. Glück, E. Reya and A. Vogt, Eur. Phys. J. C 5 (1998) 461.

[7] F.E. Close and A.W. Thomas, Phys. Lett. B 212 (1988) 227;

A.W. Thomas, Phys. Lett. B 126 (1983) 97;

R.P. Feynman, Photon-Hadron Interactions, W.A. Benjamin, New York 1972. 
[8] W. Melnitchouk and A.W. Thomas, Phys. Lett. B 377 (1996) 11.

[9] S. Kumano, Phys. Rep. 303 (1998) 183.

[10] A.W. Schreiber, P.J. Mulders, A.I. Signal and A.W. Thomas, Phys. Rev. D 45 (1992) 3069;

A. Szczurek, A. Buchmann and A. Faessler, J. Phys. G 22 (1996) 1741;

K. Suzuki and W. Weise, Nucl. Phys. A 634 (1998) 141.

[11] Y. Nambu and G. Jona-Lasinio, Phys. Rev. 122 (1960) 345; 124 (1961) 246.

[12] T. Hatsuda and T. Kunihiro, Phys. Rep. 247 (1994) 221;

U. Vogl and W. Weise, Prog. Part. Nucl. Phys. 27 (1991) 195.

[13] N. Ishii, W. Bentz and K. Yazaki, Phys. Lett. B 301 (1993) 165;

N. Ishii, W. Bentz and K. Yazaki, Phys. Lett. B 318 (1993) 26.

[14] N. Ishii, W. Bentz and K. Yazaki, Nucl. Phys. A 578 (1995) 617.

[15] U. Zückert, R. Alkofer, H. Weigel and H. Reinhardt, Phys. Rev. C 55 (1997) 2030;

S. Huang and J. Tjon, Phys. Rev. C 49 (1994) 1702;

C. Hanhart and S. Krewald, Phys. Lett. B 344 (1995) 55.

[16] N. Ishii, Nucl. Phys. A 689 (2001) 793.

[17] M. Oettel, G. Hellstern, R. Alkofer and H. Reinhardt, Phys. Rev. C 58 (1998) 2459.

[18] M. Oettel, R. Alkofer and L. von Smekal, Eur. Phys. J. A 8 (2000) 553. 
[19] W. Bentz, T. Hama, T. Matsuki, and K. Yazaki, Nucl. Phys. A 651, 143 (1999).

[20] A. Buck, R. Alkofer and H. Reinhardt, Phys. Lett. B 286 (1992) 29.

[21] H. Mineo, W. Bentz and K. Yazaki, Phys. Rev. C 60 (1999) 065201.

[22] H. Asami, N. Ishii, W. Bentz and K. Yazaki, Phys. Rev. C 52 (1996) 3388.

[23] R.L. Jaffe, 1985 Los Alamos School on Relativistic Dynamics and Quark Nuclear Physics, eds. M.B. Johnson and A. Pickleseimer, Wiley, New York, 1985;

R.L. Jaffe, Nucl. Phys. B 229 (1983) 205.

[24] G.P. Lepage and S.J. Brodsky, Phys. Rev. D 22 (1980) 2157.

[25] G. Hellstern and C. Weiss, Phys. Lett. B 351 (1995) 64.

[26] D.B. Leinweber, D.H. Lu and A.W. Thomas, Phys. Rev. D 60 (1999) 033014.

[27] V.N. Gribov and L.N. Lipatov, Sov. J. Nucl. Phys. 15 (1972) 438 and 675 ;

G. Altarelli and G. Parisi, Nucl. Phys. B 126 (1977) 298;

Yu.L. Dokshitzer, Sov. Phys. JETP 46 (1977) 641.

[28] M. Miyama and S. Kumano, Comput. Phys. Commun. 94 (1996) 185.

[29] G. R. Farrar and D. R. Jackson, Phys. Rev. Lett. 35 (1975) 1416. 
[30] S.J. Brodsky, M. Burkardt and I. Schmidt, Nucl. Phys. B 441 (1995) 197.

[31] K. Suzuki, W. Weise, Nucl. Phys. A 634 (1998) 141.

[32] A.A. Bel'kov, D. Ebert and A.V. Emelyanenko, Nucl Phys. A 552 (1993) 523 ;

K. Langfeld and M. Rho, Nucl. Phys. A 596 (1996) 451.

[33] G. Hellstern, R. Alkofer and H. Reinhardt, Nucl. Phys. A 625 (1997) 697.

[34] W. Bentz and A.W. Thomas, nucl-th/0105022, and Nucl. Phys. A (2001), in press. 


\section{Figure Captions}

1. Graphical representation of the quark LC momentum distribution in the nucleon. The single (double) line denotes the constituent quark propagator (diquark t-matrix), the hatched circle the quark-diquark vertex function, and the operator insertion stands for $\gamma^{+} \delta\left(k_{-}-p_{-} x\right)(1 \pm$ $\left.\tau_{z}\right) / 2$ for the $\mathrm{U}(\mathrm{D})$ quark distribution.

2. Diagrams representing the dressing of the constituent quark by the pion cloud. The dashed line denotes the $q \bar{q}$ t-matrix in the pionic channel.

3. Feynman diagram where the operator insertion is made on the quark exchanged between the diquark and the spectator quark. For the quark LC momentum distributions and the electromagnetic current, this diagram does not contribute in the static approximation. However, it should be considered for the calculation of $g_{\pi N N}$ as explained in the text.

4. Comparison of the valence quark distributions $x u_{v}(x) / 2$ and $x d_{v}(x)$ for the cases I (dashed lines), II (solid lines), and III (dotted lines) of Table 1.

5. Ratio of neutron to proton structure functions for several values of the ratio $r_{a}$. For each $r_{a}$, the value of $r_{s}$ is determined so as to reproduce the experimental nucleon mass. The data points, which are taken from Fig. 3 of ref. [8], are based on the SLAC proton and deuteron data using a deuteron model with on-shell nucleons (upper data points), and including binding and off-shell effects (lower data points). 
6. Valence quark distributions (multiplied by $x$ ) corresponding to case II of Table 1. The solid lines show the input distributions $\left(\mu^{2}=Q_{0}^{2}\right)$ calculated in the NJL model, the dashed lines show the distributions obtained by the QCD evolution up to $Q^{2}=4 \mathrm{GeV}^{2}$ in next-to-leading order, and the dotted lines are the empirical distributions of ref. [6].

7. Same as Fig. 6 for the sea quark distributions.

8. Results for the proton structure function $F_{2}^{p}(x)$ at $Q^{2}=4.5 \mathrm{GeV}^{2}$ (a) and $Q^{2}=15 \mathrm{GeV}^{2}$ (b) for case II of Table 1 , in comparison with the experimental data of ref. [3].

9. The magnetic moment of the proton (a) and the neutron (b) as functions of $r_{a}$. The dashed lines are calculated in the valence quark picture, while the solid lines include the effects of the pion cloud as described in the text. For each $r_{a}$, the value of $r_{s}$ is determined so as to reproduce the experimental nucleon mass.

10. Same as Fig. 9 for the isovector (a) and the isoscalar (b) axial vector coupling constants of the nucleon.

11. Same as Fig. 9 for the $\pi N N$ coupling constant (a) and the ratio $\Delta_{G T}=$ $f_{\pi} g_{\pi N N} / M_{N} g_{A}^{(3)}(\mathrm{b})$. For $\Delta_{G T}$ we also show the result obtained by subtracting the contact terms of the t-matrices in the scalar and a.v. diquark channels, as explained in the text.

12. The axial vector vertex of the constituent quark including the pion pole terms. The bubbles graphs here involve $q \bar{q}$ states in the pionic channel. 


\section{Appendices}

\section{A Diagonalization of the Faddeev kernel in the static approximation}

In this Appendix we directly diagonalize the Faddeev kernel in the static approximation (see eq.(2.9) for the $T=\frac{1}{2}$ states) and derive the form of the nucleon vertex function as given in eqs. (2.13)-(2.15). The diagonalization will be done in the rest frame of the nucleon $\left(p^{\mu}=\left(M_{N}, \mathbf{0}\right)\right)$, and the solution will be boosted to a general frame.

In order to remove the Dirac $\gamma$ matrices from the quark exchange kernel $Z$ (eq.(2.10) ), we first apply the following unitary transformation:

$$
\tilde{K}(p)=\Omega K(p) \Omega^{\dagger} \equiv \tilde{Z} \tilde{\Pi}_{N}(p)
$$

where $\Omega=\operatorname{diag}\left(\gamma_{5}, \gamma^{\mu}\right)$ and $\tilde{Z}=\Omega Z \Omega^{\dagger}, \tilde{\Pi}_{N}(p)=\Omega \Pi_{N}(p) \Omega^{\dagger}$. $\tilde{Z}$ has the form

$$
\tilde{Z}=\frac{3}{M}\left(\begin{array}{ccc}
1 & -\sqrt{3} & -\sqrt{3} x^{T} \\
-\sqrt{3} & -1 & x^{T} \\
-\sqrt{3} x & x & X
\end{array}\right), \quad \text { where } X=\left(\begin{array}{ccc}
-1 & 1 & 1 \\
1 & -1 & 1 \\
1 & 1 & -1
\end{array}\right)
$$

and $x^{T}=(1,1,1)$. In the rest frame of the nucleon, the transformed quarkdiquark bubble graph $\tilde{\Pi}_{N}(p)$ involves only the Dirac matrices 1 and $\gamma_{0}$, and can be written as follows:

$$
\begin{aligned}
\tilde{\Pi}_{N}(p) & =\tilde{G}_{+}(p) \frac{1-\gamma_{0}}{2}+\tilde{G}_{-}(p) \frac{1+\gamma_{0}}{2} \\
& \equiv \tilde{\Pi}_{N(+)}(p)+\tilde{\Pi}_{N(-)}(p)
\end{aligned}
$$


with

$$
G_{+}(p)=\left(\begin{array}{ccc}
a & 0 & 0 \\
0 & b & c x^{T} \\
0 & c x & d I
\end{array}\right), \quad G_{-}(p)=(),
$$

where $I=\operatorname{diag}(1,1,1)$ and

$$
\begin{aligned}
a(p) & =\int \frac{d^{4} q}{(2 \pi)^{4}} \frac{\left(p_{0}-q_{0}\right)+M}{(p-q)^{2}-M^{2}} \tau_{s}(q) \\
b(p) & =\int \frac{d^{4} q}{(2 \pi)^{4}} \frac{-\left(p_{0}-q_{0}\right)+M}{(p-q)^{2}-M^{2}} \tau_{a}^{00}(q) \\
c(p) & =\int \frac{d^{4} q}{(2 \pi)^{4}} \frac{q^{i}}{(p-q)^{2}-M^{2}} \tau_{a}^{0 i}(q) \quad \text { (no sum) } \\
d(p) & =\int \frac{d^{4} q}{(2 \pi)^{4}} \frac{\left(p_{0}-q_{0}\right)+M}{(p-q)^{2}-M^{2}} \tau_{a}^{i i}(q) \quad \text { (no sum). }
\end{aligned}
$$

In eqs. (A.8) and (A.9) the index $i$ is fixed to be any among $i=1,2,3$, i.e.; one can replace $q^{i} q^{i} \rightarrow \boldsymbol{q}^{2} / 3$ in these relations. The quantity $\tilde{\Pi}_{N(+)}(p)$ in (A.4) gives the positive parity part of the transformed kernel, while $\tilde{\Pi}_{N(-)}(p)$ refers to the negative parity In the following, we will refer only to the positive parity part without changing the notation, i.e, $\tilde{\Pi}_{N} \equiv \tilde{\Pi}_{N(+)}, \tilde{K} \equiv \tilde{Z} \tilde{\Pi}_{N(+)}(p)$, etc. 10 .

To the transformed eigenvalue equation $\tilde{\Gamma}(p)=\tilde{K}(p) \tilde{\Gamma}(p)$, we apply a further orthogonal transformation in order to bring the kernel into a block form corresponding to $J=\frac{1}{2}$ and $J=\frac{3}{2}$ states:

$$
\hat{K}(p)=U \tilde{K}(p) U^{t} \equiv \hat{Z} \hat{\Pi}_{N}(p)
$$

\footnotetext{
${ }^{16}$ The reason is as follows: In the rest frame of the nucleon the projection operator onto positive parity is (in the original representation) $P_{+}=\operatorname{diag}\left(p_{+}, p_{-}, p_{+}, p_{+}, p_{+}\right)$, where $p_{ \pm}=\left(1 \pm \gamma_{0}\right) / 2$, and the diagonal matrix refers to the 5 diquark indices $a=5$ (scalar diquark), $a=0$ (time component of the a.v. diquark) and $a=1,2,3$ (space components of the a.v. diquark). After applying the unitary transformation $\Omega=\operatorname{diag}\left(\gamma_{5}, \gamma^{\mu}\right)$ as in (A.1), this becomes $\tilde{P}_{+}=\operatorname{diag}\left(p_{-}, p_{-}, p_{-}, p_{-}, p_{-}\right)$.
} 
with $U=\operatorname{diag}(1,1, Y)$ and the $3 \times 3$ matrix $Y$ diagonalizes $X$ of eq.(A.2):

$$
Y=\left(\begin{array}{ccc}
1 / \sqrt{3} & 0 & -2 / \sqrt{6} \\
1 / \sqrt{3} & -1 / \sqrt{2} & 1 / \sqrt{6} \\
1 / \sqrt{3} & 1 / \sqrt{2} & \sqrt{6} / 3
\end{array}\right)
$$

The transformed quantities $\hat{Z}$ and $\hat{\Pi}_{N}(p)$ are given by the block forms

$$
\begin{gathered}
\hat{Z}=\frac{3}{M}\left(\begin{array}{ccccc}
1 & -\sqrt{3} & -3 & & \\
-\sqrt{3} & -1 & \sqrt{3} & & \\
-3 & \sqrt{3} & 1 & & \\
& & & -2 & \\
& & & & -2
\end{array}\right), \\
\hat{\Pi}_{N}(p)=\left(\begin{array}{ccccc}
a & & & & \\
b & \sqrt{3} c & & \\
\sqrt{3} c & d & & \\
& & & d & \\
& & & & d
\end{array}\right)
\end{gathered}
$$

and the eigenvalue equation $\hat{\Gamma}(p)=\hat{Z} \hat{\Pi}_{N}(p) \hat{\Gamma}(p)$ separates into two simple equations, one with dimension $3 \times 2=6$ and one with $2 \times 2=4$. The latter one clearly corresponds to $J=\frac{3}{2}$, and the former one to $J=\frac{1}{2}$, since there are 3 basis states corresponding to (i) the coupling of a scalar diquark and a quark, (ii) the time component of the a.v. diquark and a quark, and (iii) the space components of the a.v. diquark and a quark, and each of these three states has two spin directions.

The solutions for the nucleon vertex function in this representation therefore have the form

$$
\hat{\Pi}_{N \uparrow}=\left(\begin{array}{c}
\alpha_{1} \\
\alpha_{2} \\
\alpha_{3} \\
0 \\
0
\end{array}\right)_{\text {diquark }} \bigotimes\left(\begin{array}{l}
0 \\
0 \\
1 \\
0
\end{array}\right)_{\text {quark }}, \quad \hat{\Pi}_{N \downarrow}=\left(\begin{array}{c}
\alpha_{1} \\
\alpha_{2} \\
\alpha_{3} \\
0 \\
0
\end{array}\right)_{\text {diquark }} \bigotimes\left(\begin{array}{l}
0 \\
0 \\
0 \\
1
\end{array}\right)_{\text {quark }}
$$


corresponding to the two spin projections, where $\left(\alpha_{1}, \alpha_{2}, \alpha_{3}\right)$ is the eigenvector of the upper $3 \times 3$ block of $\hat{K}$ in (A.10) with the largest eigenvalue $\lambda_{N}(p)$. The nucleon mass is then determined by $\lambda_{N}\left(p_{0}=M_{N}\right)=1$.

It is then easy to transform the solutions (A.14) back to the original representation using $\Pi_{N}(p)=\Gamma^{\dagger} U \hat{\Pi}_{N}(p)$, and then to apply a boost which is the product of a Lorentz transformation for spinors and ordinary 4-vectors, respectively, both with velocity $\boldsymbol{v}=-\boldsymbol{p} / E_{N}(p)$ with $E_{N}(p)=\sqrt{M_{N}^{2}+\boldsymbol{p}^{2}}$. The result is given by eqs. (2.13), (2.14), where

$$
\epsilon_{\lambda}^{\mu}(p)=\left(\frac{\boldsymbol{p} \cdot \boldsymbol{\epsilon}_{\lambda}}{M_{N}}, \boldsymbol{\epsilon}_{\lambda}+\frac{\boldsymbol{p}\left(\boldsymbol{p} \cdot \boldsymbol{\epsilon}_{\lambda}\right)}{M_{N}\left(E_{N}(p)+M_{N}\right)}\right),
$$

where $\boldsymbol{\epsilon}_{\lambda}$ are the usual spherical unit vectors.

To derive the covariant form (2.15) from (2.14), one makes use of the relations

$$
\begin{aligned}
\left(1 \frac{1}{2}, \lambda s^{\prime} \mid \frac{1}{2} s\right) & =\frac{(-1)^{\lambda+1}}{\sqrt{3}}\left(\sigma_{-\lambda}^{[1]}\right)_{s^{\prime} s} \\
\sum_{s^{\prime}}(\boldsymbol{\sigma} \cdot \boldsymbol{p})_{s^{\prime} s} \chi_{s^{\prime}} & =(\boldsymbol{\sigma} \cdot \boldsymbol{p}) \chi_{s},
\end{aligned}
$$

where (A.16) follows from the Wigner-Eckert theorem, and $\chi$ in (A.17) is a 2-component Pauli spinor.

The diagonalization of the $T=\frac{3}{2}$ kernel of eq.(2.17) proceeds in the same way by applying the unitary transformations $\Gamma$ and $U$ as above. The positive parity part of the kernel then separates into a $2 \times 2$ block for $J=\frac{1}{2}$ (corresponding to the coupling of the time component and the space components of the a.v. diquark with the quark, respectively) and a diagonal $2 \times 2$ block for $J=\frac{3}{2}$. The latter one has the same form as the lower $2 \times 2$ block of the kernel (A.10), but with the factor -2 in (A.12) replaced by 4 . The delta 
mass is therefore determined by the equation $\frac{12}{M} d\left(p_{0}=M_{\Delta}\right)=1$, and after transforming back to the original representation and boosting the vertex function has the form of a Rarita-Schwinger spinor:

$$
\Gamma_{\Delta}^{\mu}(p)=b \sum_{\lambda s^{\prime}}\left(1 \frac{1}{2}, \lambda s^{\prime} \mid \frac{3}{2}\right) \epsilon_{\lambda}^{\mu}(p) u_{N}\left(p, s^{\prime}\right),
$$

where $b$ is a normalization constant. 


\section{B PCAC in the Faddeev framework}

In this Appendix we demonstrate the validity of the PCAC relation for the case of the scalar diquark channel only, both in the exact Faddeev framework and in the static approximation. For more detailed discussions we refer to ref. [16.

In the exact Faddeev framework, the isovector axial vector current of the nucleon is given by the sum of the 'quark diagram' and the 'exchange diagram' (see fig.3) as follows:

$$
\begin{aligned}
j_{A}^{\mu}(q) & =\int \frac{\mathrm{d}^{4} k}{(2 \pi)^{4}} \int \frac{\mathrm{d}^{4} k^{\prime}}{(2 \pi)^{4}} \bar{\Gamma}_{N, P^{\prime}}\left(k^{\prime}\right) \\
& \times\left[S_{F}\left(\frac{P}{2}+k^{\prime}\right) \Gamma_{q}^{\mu} S_{F}\left(\frac{P}{2}+k\right) \tau_{s}\left(\frac{P}{2}-k\right) \delta\left(k^{\prime}-k-\frac{q}{2}\right)\right. \\
& +3 S_{F}\left(\frac{P^{\prime}}{2}+k^{\prime}\right) \gamma_{5} F_{F}\left(k+k^{\prime}+\frac{q}{2}\right) \Gamma_{q}^{\mu} S_{F}\left(k+k^{\prime}-\frac{q}{2}\right) \gamma_{5} S_{F}\left(\frac{P}{2}+k\right) \\
& \left.\times \tau_{s}\left(\frac{P^{\prime}}{2}-k^{\prime}\right) \tau_{s}\left(\frac{P}{2}-k\right)\right] \Gamma_{N, P}(k)
\end{aligned}
$$

Here the assignments of the momenta are as in refs. [14, 22], and the matrices $C \tau_{2}$, which appear in the 2-body vertex functions in the exchange diagram, have been processed as explained in ref. [22]. The Faddeev equation for the nucleon spinor reads

$$
\Gamma_{N, P}(p)=\int \frac{\mathrm{d}^{4} p^{\prime}}{Z}\left(p, p^{\prime}\right) S_{F}\left(\frac{P}{2}+p^{\prime}\right) \tau_{s}\left(\frac{P}{2}-p^{\prime}\right) \Gamma_{N, P}\left(p^{\prime}\right)
$$

with the quark exchange kernel $Z\left(p, p^{\prime}\right)=-3 \gamma_{5} S_{F}\left(p^{\prime}+p\right) \gamma_{5}$. The quark axial vector vertex $\Gamma_{q}^{\mu}$ including the vertex corrections describing the pion pole is given by (see fig. 12)

$$
\Gamma_{q}^{\mu}(q)=\gamma^{\mu} \gamma_{5} \tau_{3}-i \Pi^{\mu}(q) \tau_{3} \gamma_{5} \tau_{\pi}(q)
$$


where $\Pi^{\mu}(q)$ is the bubble graph describing the pion-axial vector mixing:

$$
\Pi^{\mu}(q)=12 i M q^{\mu} \int \frac{\mathrm{d}^{4} k}{(2 \pi)^{4}} \frac{1}{\left(k^{2}-M^{2}+i \epsilon\right)\left((k+q)^{2}-M^{2}+i \epsilon\right)}
$$

Using the definition of $f_{\pi}$, and defining the 'physical' pion propagator by $\Delta_{\pi}(q) \equiv \frac{-i}{g^{2}} \tau_{\pi}$ so that its pole term is simply given by $\frac{1}{q^{2}-m_{\pi}^{2}}$ with unit residue, it is easy to show that the vertex (B.4) satisfies the axial WardTakahashi identity of the linear sigma model:

$$
q_{\mu} \Gamma_{q}^{\mu}(q)=\tau_{3}\left(S^{-1}\left(p^{\prime}\right) \gamma_{5}+\gamma_{5} S^{-1}(p)\right)-2 c \Delta_{\pi}(q) \Gamma_{q \pi}
$$

with $\Gamma_{q \pi}=\tau_{3} \gamma_{5} g$ and $c=f_{\pi} m_{\pi}^{2}$. We also note that if the pion pole terms are removed from the beginning, as is sufficient for the calculation of $g_{A}$ (i.e., if only the first term in fig. 12 is taken for the quark axial vector vertex), the corresponding axial Ward-Takahashi identity is obtained from (B.6) by the replacement $-c \Delta_{\pi}(q) \rightarrow \frac{M}{g}$, which is equal to $f_{\pi}$. Using (B.6) in (B.2) and using the Faddeev equation (B.3) together with the corresponding equation for $\bar{\Gamma}_{N, p^{\prime}}$, it is easy to derive the PCAC relation

$$
q_{\mu} j_{A}^{\mu}(q)=-2 c \Delta_{\pi}(q) j_{\pi}(q)
$$

where the pion absorption current is given by $(B .2)$ with the replacement $\Gamma_{q}^{\mu} \rightarrow \Gamma_{\pi q}$

According to the discussions in sect. 4.2, in the static approximation the axial current is given by the quark diagram, while for the pion absorption current we have to consider also the exchange diagram (fig. 3). The expressions are as follows (see sect. 4.2):

$$
j_{A}^{\mu}(q)=\bar{\Gamma}_{N, P^{\prime}} \int \frac{\mathrm{d}^{4} k}{(2 \pi)^{4}} S_{F}(k+q) \Gamma_{q}^{\mu} S_{F}(k) \tau_{s}(p-k) \Gamma_{N, P}
$$




$$
\begin{aligned}
j_{\pi}(q) & =\bar{\Gamma}_{N, P^{\prime}} \int \frac{\mathrm{d}^{4} k}{(2 \pi)^{4}} S_{F}(k+q) \Gamma_{\pi q} S_{F}(k) \tau_{s}(p-k) \Gamma_{N, P} \\
& +\frac{3}{M^{2}} \bar{\Gamma}_{N, P^{\prime}} 2 \Pi_{N}\left(P^{\prime}\right) \Gamma_{\pi q} \Pi_{N}(P) \Gamma_{N, P},
\end{aligned}
$$

where the quark-diquark bubble graph is given by (see eq.(2.11)

$$
\Pi_{N}(p)=-\int \frac{\mathrm{d}^{4} k}{(2 \pi)^{4}} S_{F}(p-k) \tau_{s}(k)
$$

The Faddeev equation in the static approximation reduces to (see eq. (2.9)

$$
\Pi_{N}(p) \Gamma_{N, P}=-\frac{M}{3} \Gamma_{N, P}
$$

and using $(\mathrm{B} .6),(\mathrm{B} .11)$ to calculate the divergence of the axial current $(\mathrm{B} .8)$ we arrive at the PCAC relation $(\mathbb{B} .7)$ in the static approximation. 


\begin{tabular}{|c||c|c|c||c|}
\hline case & I & II & III & $\exp$ \\
\hline$r_{a}$ & 0 & 0.25 & 0.66 & \\
\hline$r_{s}$ & 0.73 & 0.63 & 0.50 & \\
\hline$M_{s}[\mathrm{MeV}]$ & 596 & 684 & 766 & \\
\hline$W_{s}[\%]$ & 100 & 93 & 61 & \\
\hline \hline$\mu_{p}$ & 2.32 & 2.87 & 2.96 & 2.79 \\
\hline$\mu_{n}$ & -1.39 & -2.08 & -2.62 & -1.91 \\
\hline$g_{A}^{(3)}$ & 0.66 & 0.76 & 0.81 & 1.26 \\
\hline$g_{\pi \mathrm{NN}}$ & 7.5 & 12.82 & 15.34 & 13.2 \\
\hline$g_{A}^{(0)}$ & 0.60 & 0.41 & 0.30 & $0.2 \sim 0.3$ \\
\hline
\end{tabular}

Table 1: The upper part of the table shows the diquark mass $M_{s}$ and the contribution of the scalar diquark channel to the baryon number $\left(W_{s}\right)$ for three different choices of the ratio $r_{a}$. For each case, the corresponding value of $r_{s}$ is determined so as to reproduce the experimental nucleon mass. The lower part of the table shows the static properties of the nucleon obtained for these 3 cases in comparison to the experimental values. 


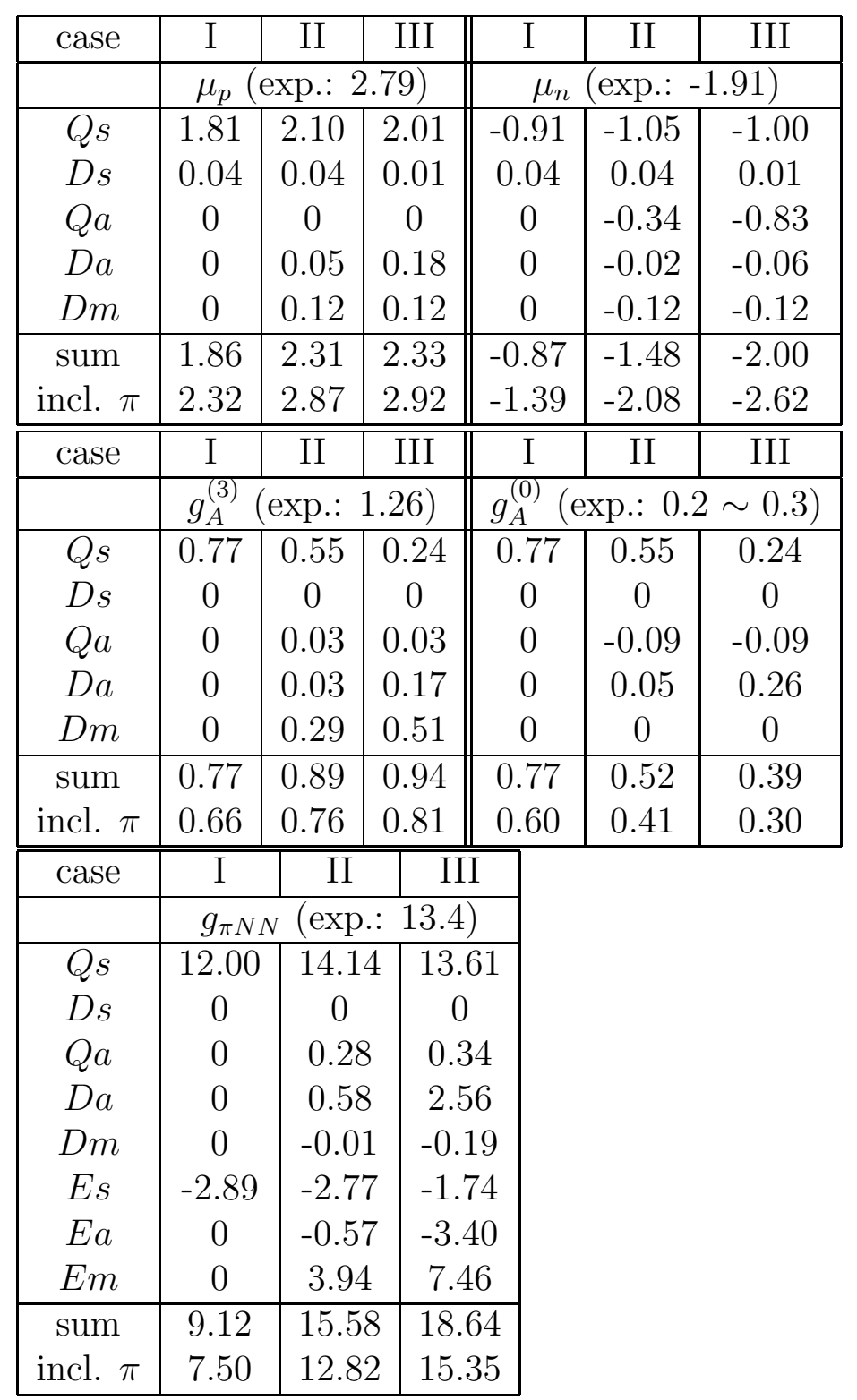

Table 2: Static properties of the nucleon for the three cases I, II, III of table 1. Qs $\left(D_{s}\right)$ denotes the contribution of the quark (diquark) diagram in the scalar channel, $Q a\left(D_{a}\right)$ refer to the quark (diquark) diagram in the a.v. channel, and $D m$ denotes the scalar-a.v. mixing in the diquark diagram. For $g_{\pi N N}$ there are in addition the exchange diagram contributions between the scalar channels $(E s)$, between the a.v. channels $(E a)$, and the mixing term $(E m)$. The sum of these contributions, as well as the total sum including pion cloud effects, are shown. 


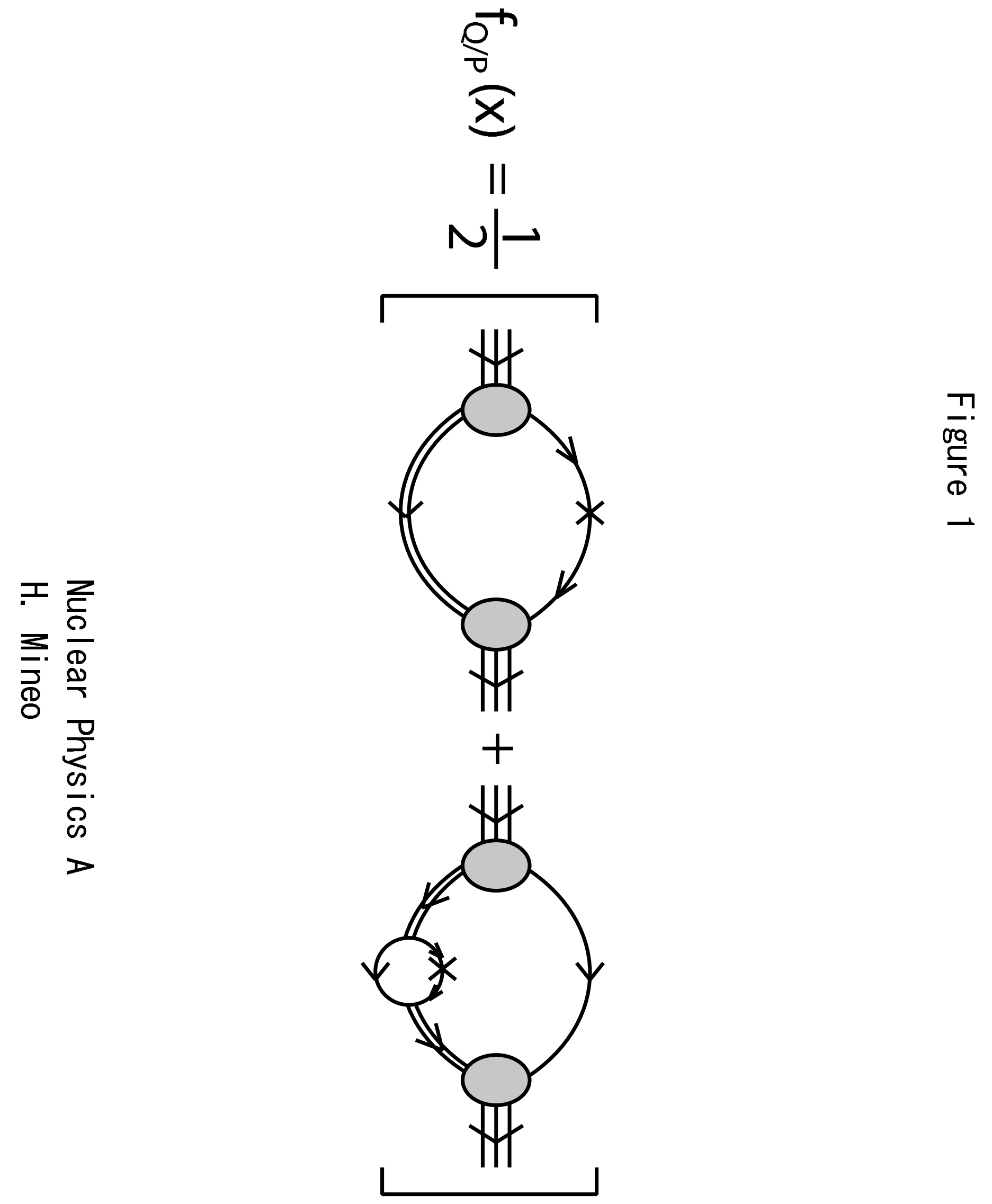




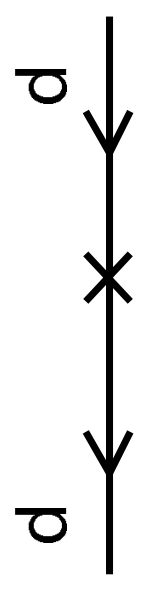

$+$
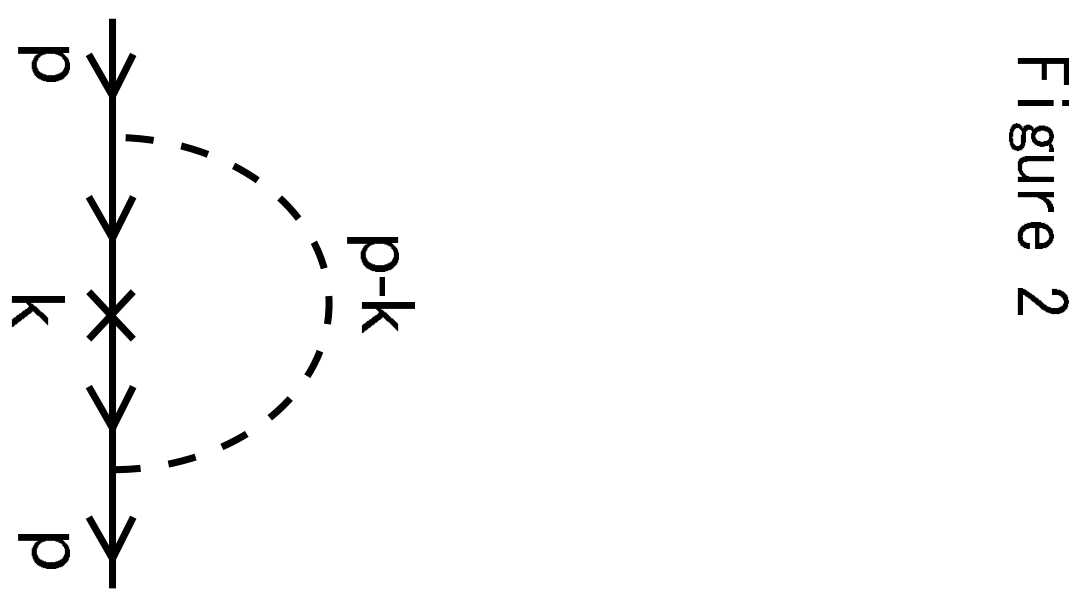

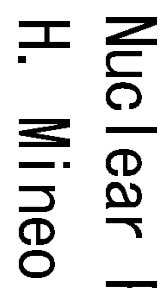

$\frac{0}{2}$
$\frac{1}{8}$
$\infty$

$+$

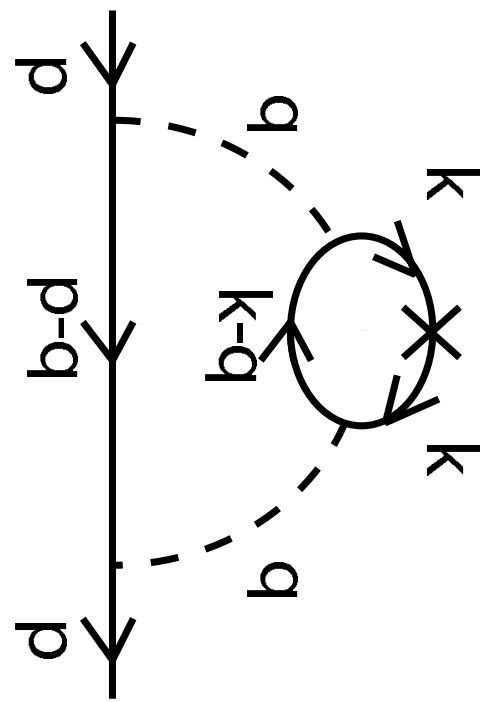




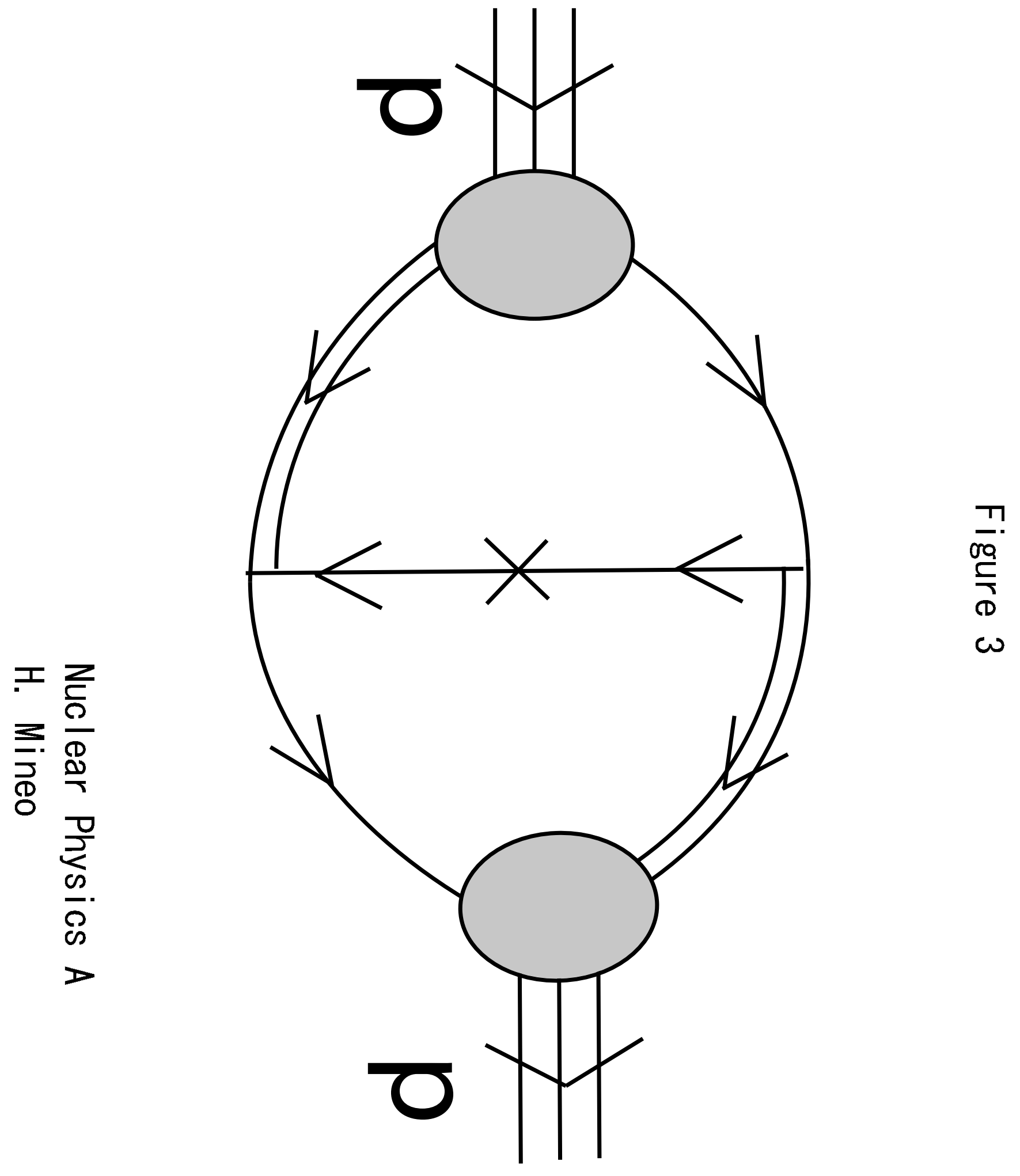




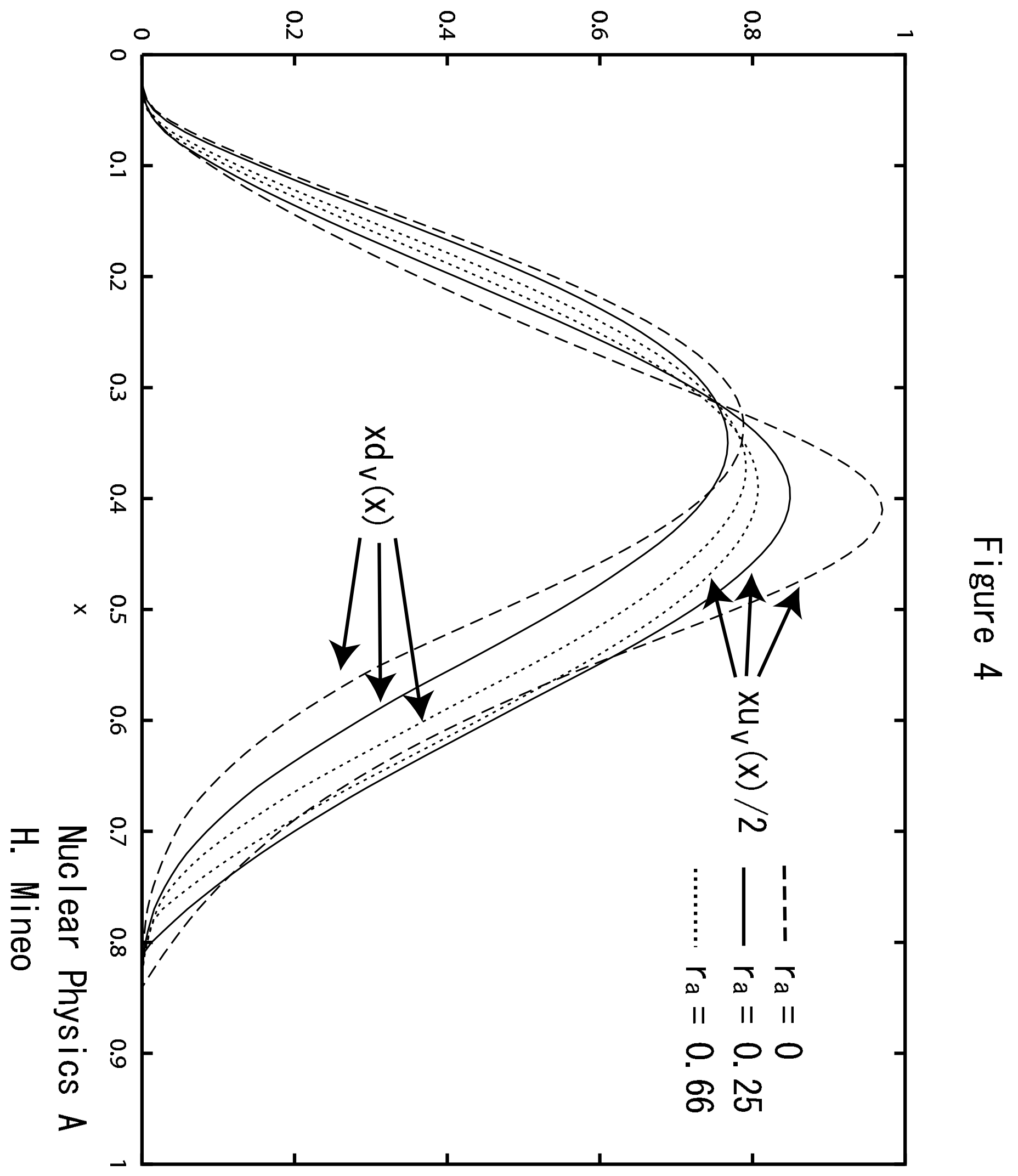




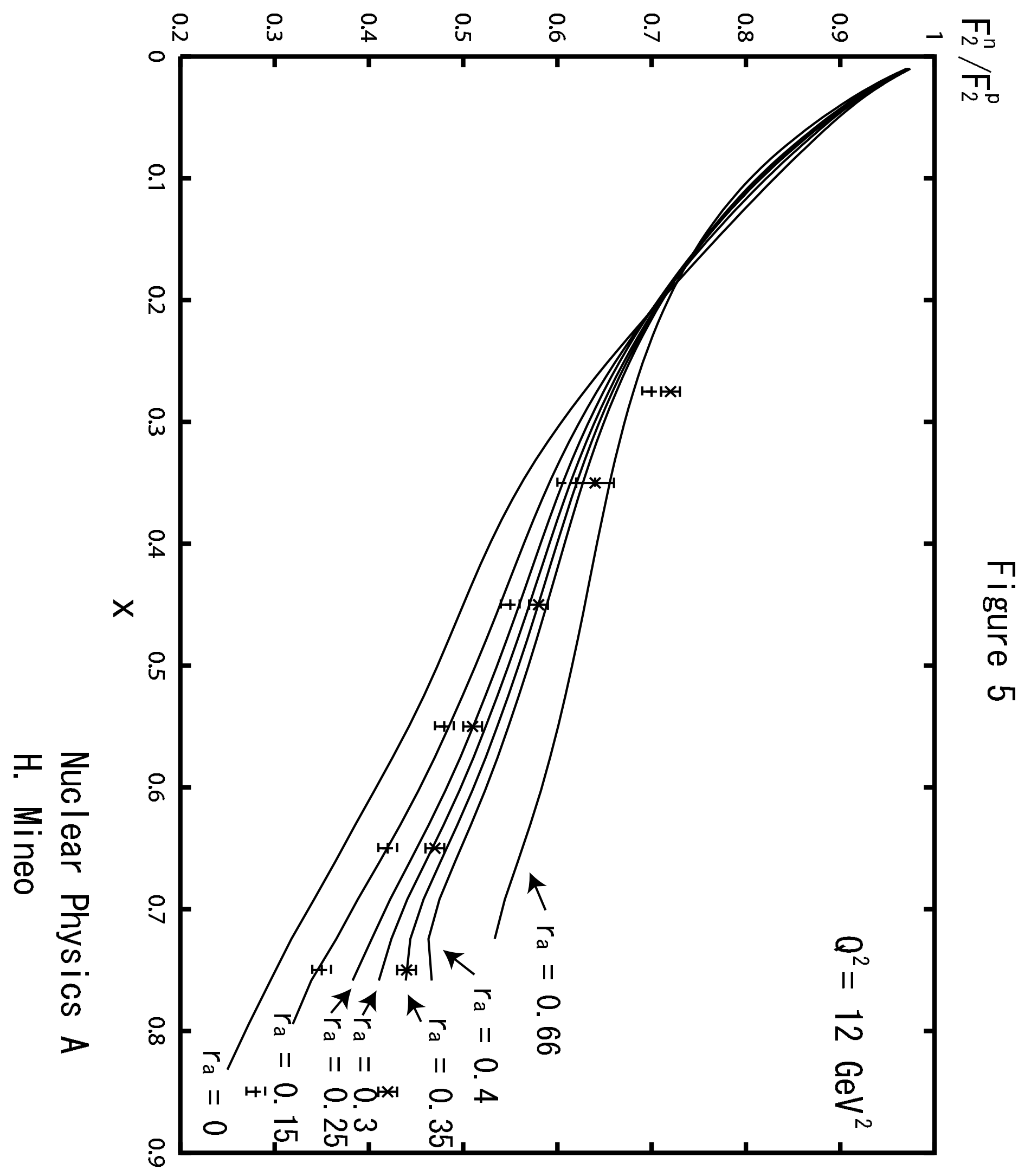


Valence quark distributions

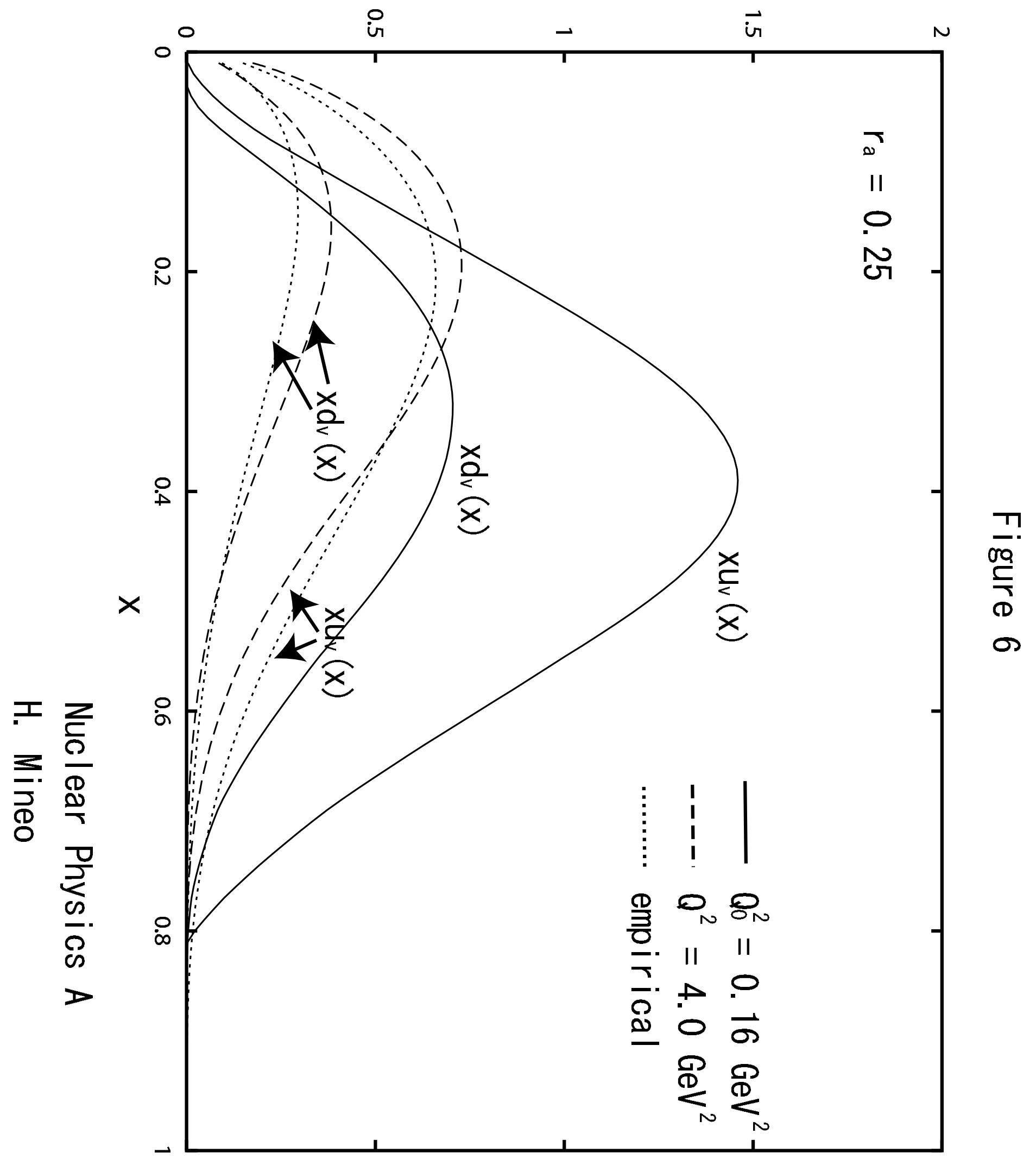


Sea quark distributions

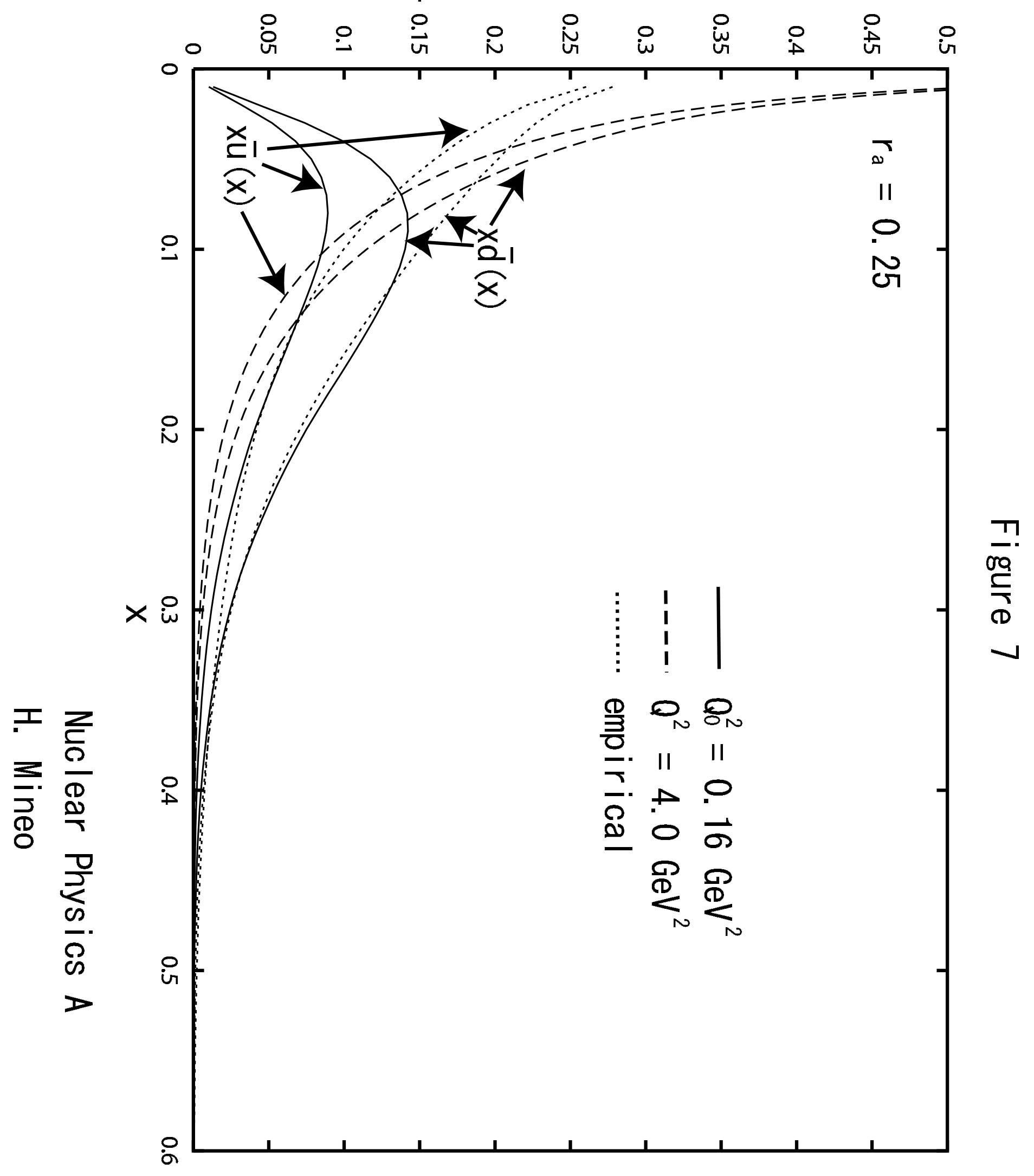




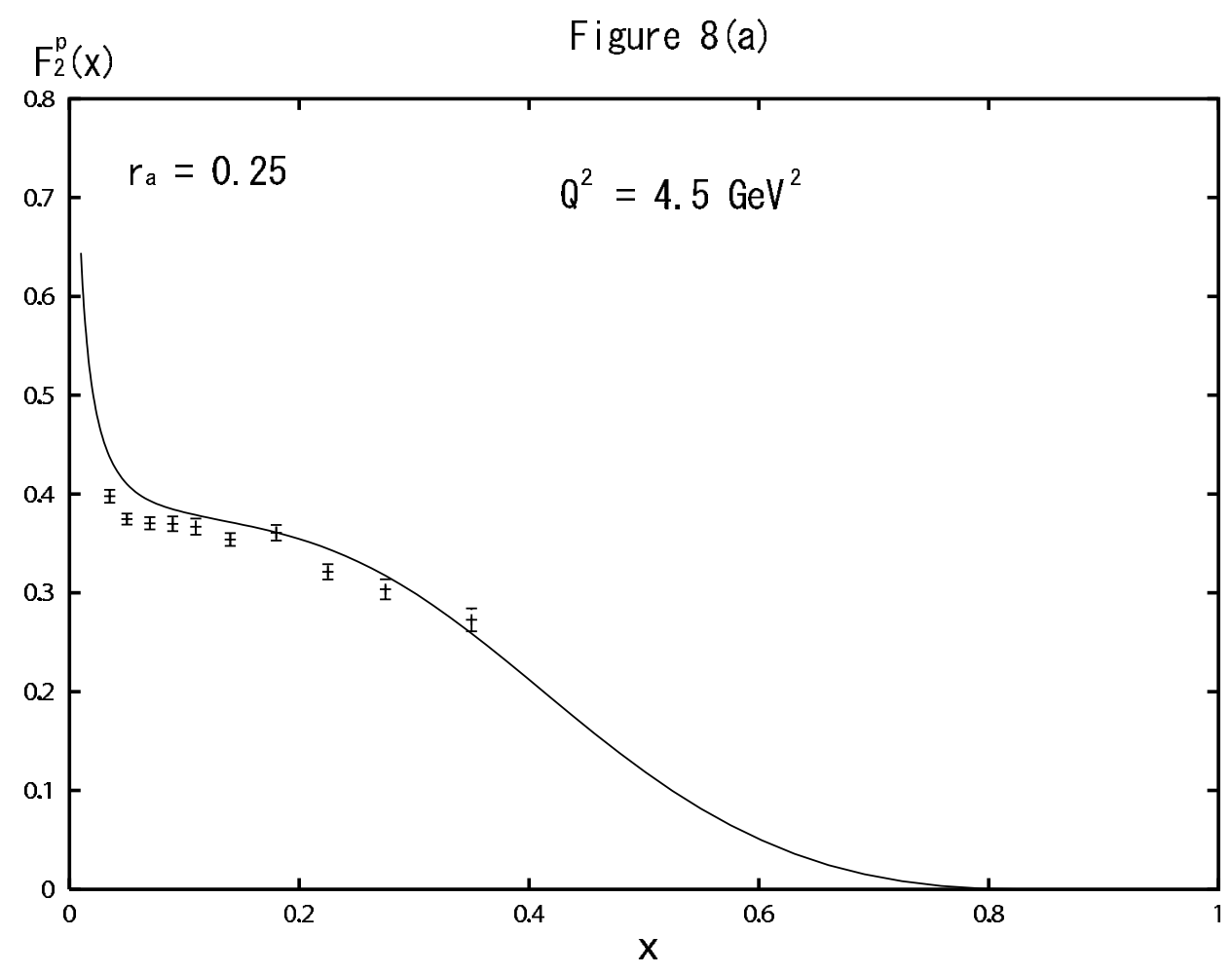

Figure $8(b)$

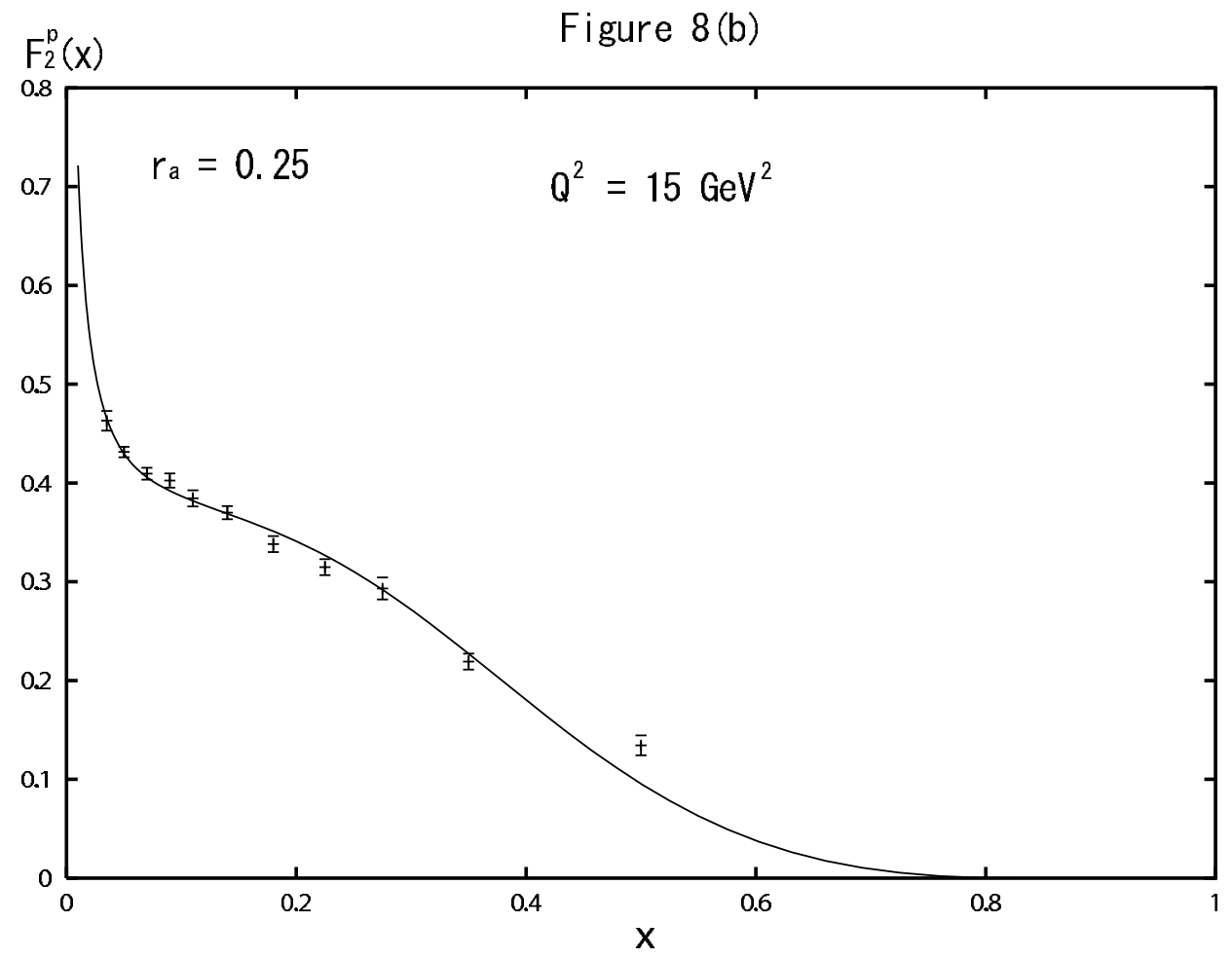

Nuclear Physics A

H. Mineo 
Figure 9(a)
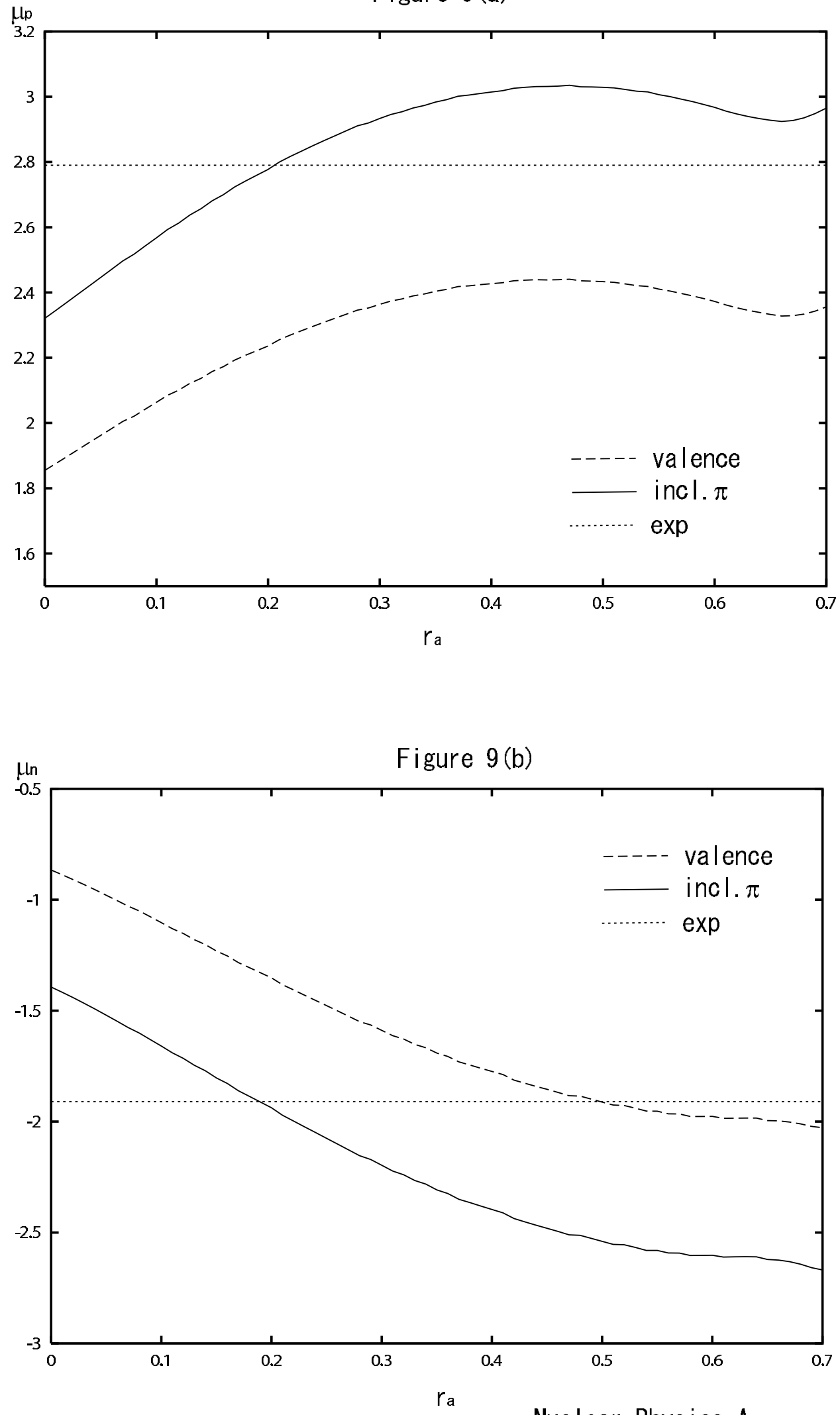

Nuclear Physics A

H. Mineo 

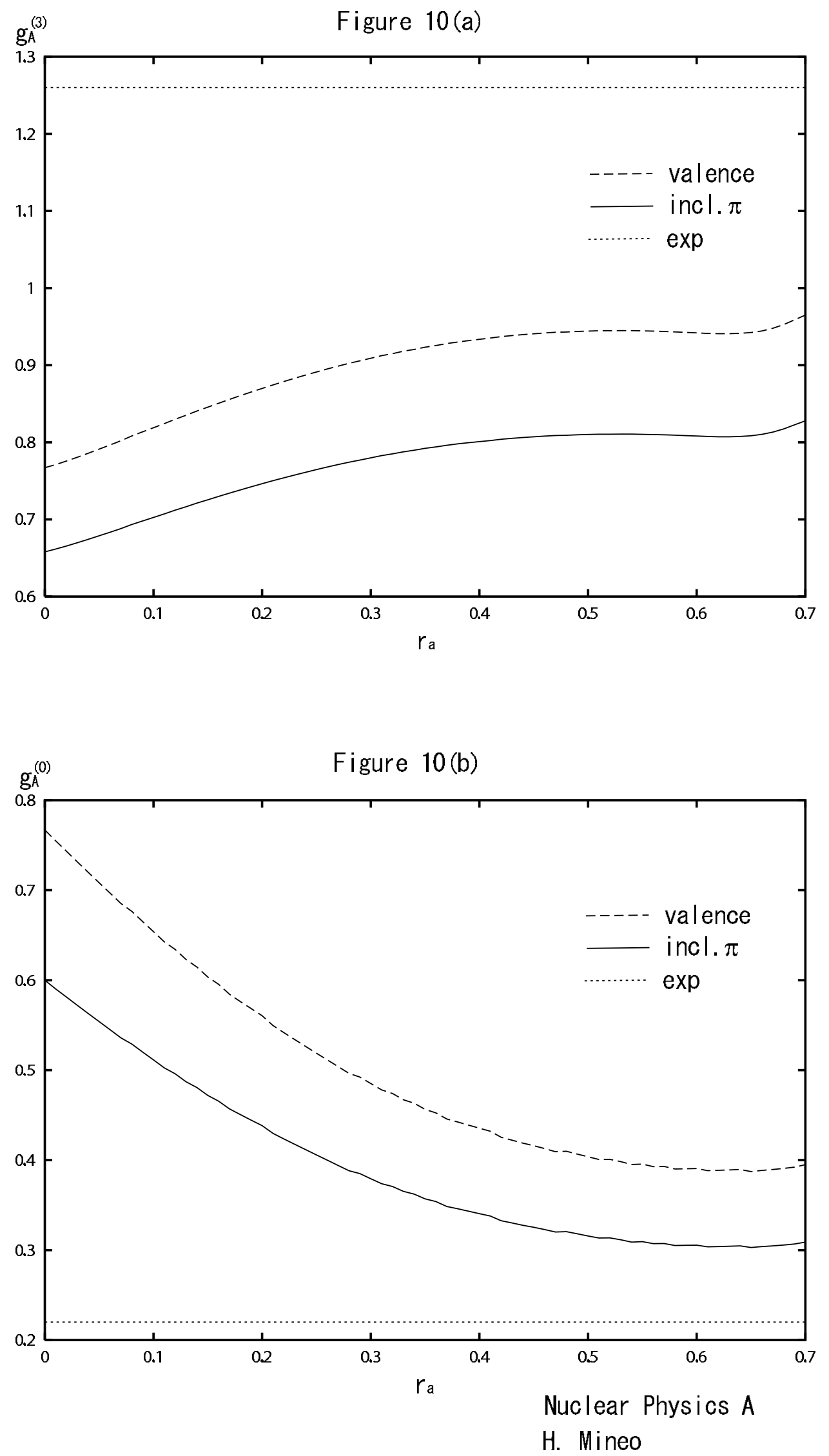

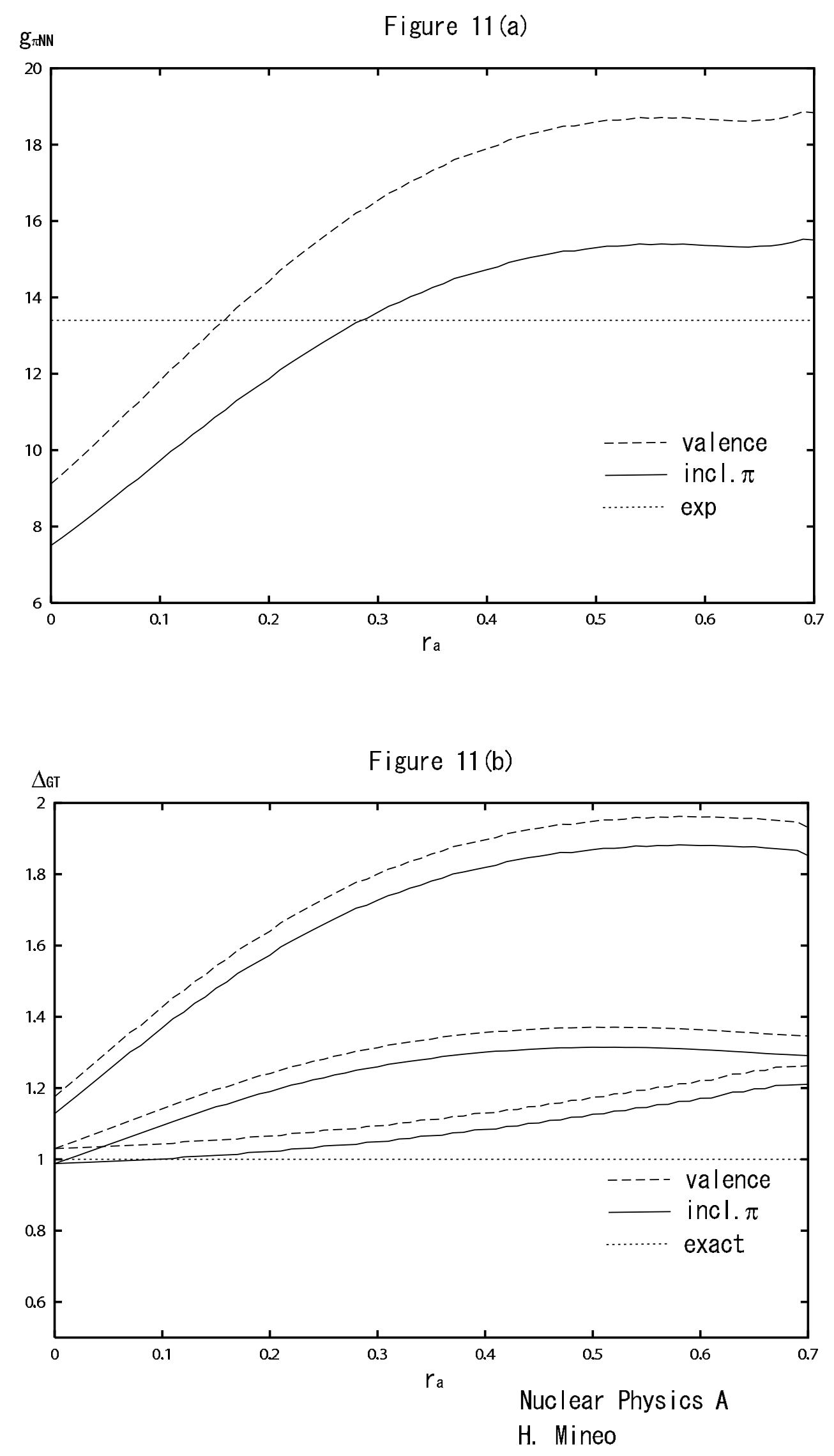


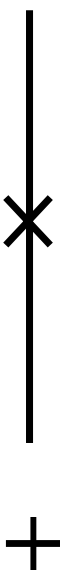

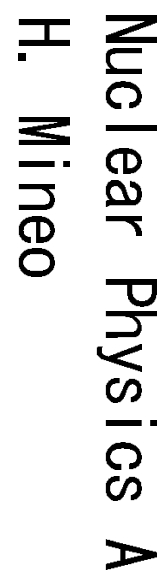
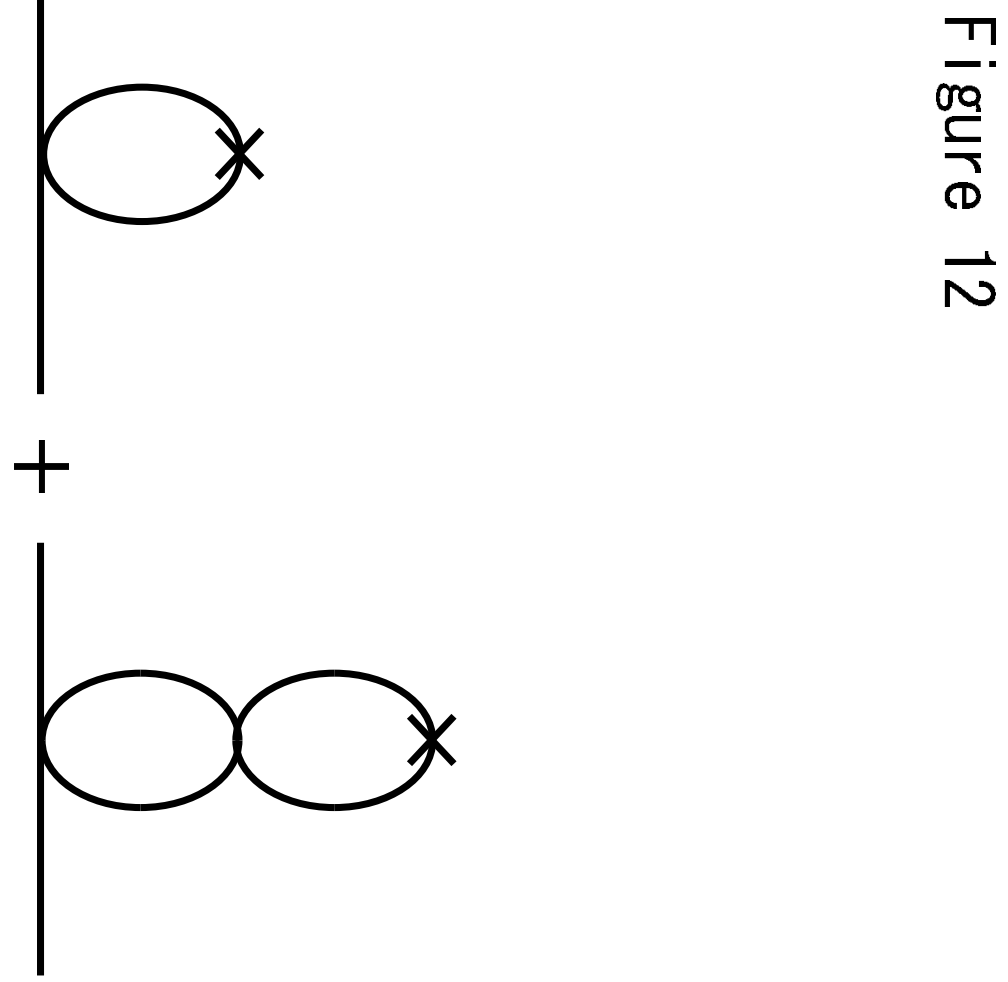

$+$

-

' 